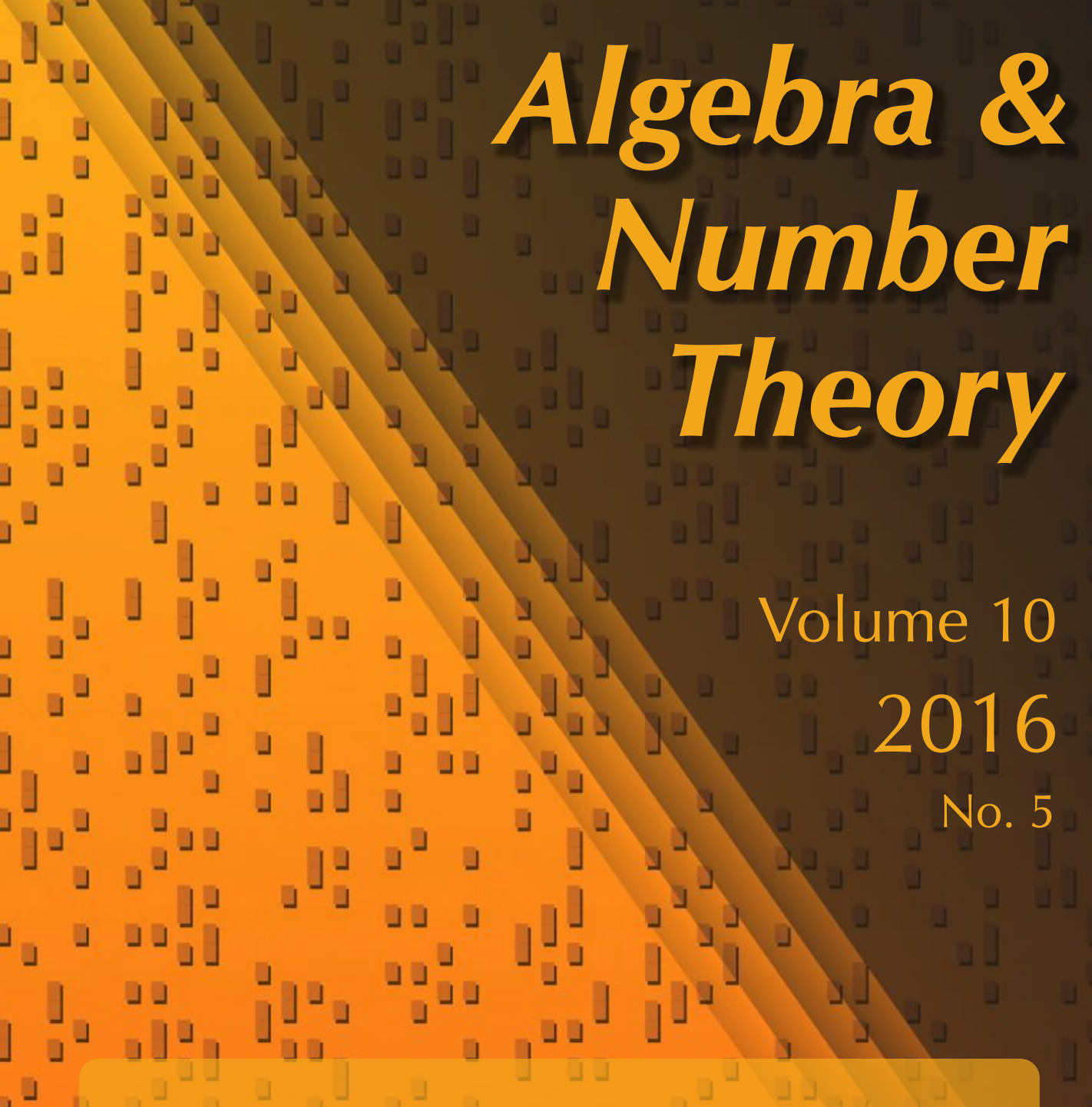

Hoffmann's conjecture for totally singular forms of prime degree

\lrcorner$\quad$ Stephen Scully

J J

ل 


\title{
Hoffmann's conjecture for totally singular forms of prime degree
}

\author{
Stephen Scully
}

\begin{abstract}
One of the most significant discrete invariants of a quadratic form $\phi$ over a field $k$ is its (full) splitting pattern, a finite sequence of integers which describes the possible isotropy behavior of $\phi$ under scalar extension to arbitrary overfields of $k$. A similarly important but more accessible variant of this notion is that of the Knebusch splitting pattern of $\phi$, which captures the isotropy behavior of $\phi$ as one passes over a certain prescribed tower of $k$-overfields. We determine all possible values of this latter invariant in the case where $\phi$ is totally singular. This includes an extension of Karpenko's theorem (formerly Hoffmann's conjecture) on the possible values of the first Witt index to the totally singular case. Contrary to the existing approaches to this problem (in the nonsingular case), our results are achieved by means of a new structural result on the higher anisotropic kernels of totally singular quadratic forms. Moreover, the methods used here readily generalize to give analogous results for arbitrary Fermat-type forms of degree $p$ over fields of characteristic $p>0$.
\end{abstract}

\section{Introduction}

Let $k$ be a field, let $\phi$ be a nonzero quadratic form on a (nonzero) $k$-vector space $V$ of finite dimension and let $X_{\phi} \subseteq \mathbb{P}(V)$ denote the projective $k$-scheme defined by the vanishing of $\phi$. Given a $k$-linear subspace $W$ of $V$, we write $\left.\phi\right|_{W}$ for the form obtained by restricting $\phi$ to $W$. In the case where $\left.\phi\right|_{W}$ is the zero form, $W$ is said to be totally isotropic (with respect to $\phi$ ). The largest integer among the dimensions of all totally isotropic subspaces of $V$ is called the isotropy index of $\phi$, and is denoted by $\mathfrak{i}_{0}(\phi)$. In the special case where $\phi$ is nonsingular (i.e., where $X_{\phi}$ is smooth), $\mathfrak{i}_{0}(\phi)$ is more commonly known as the Witt index of $\phi$, and is bounded from above by the integer part of $\frac{1}{2} \operatorname{dim} \phi$ (where $\operatorname{dim} \phi$ denotes the dimension of the $k$-vector space $V$ ). In the opposite extreme where $\phi$ is totally singular (i.e., where $X_{\phi}$ has no smooth points at all), $\mathfrak{i}_{0}(\phi)$ may take any value between 0 and $\operatorname{dim} \phi-1$.

Assume now that $\phi$ is anisotropic (i.e., that $\mathfrak{i}_{0}(\phi)=0$ ). A simple, yet fundamentally important invariant of $\phi$ is its (full) splitting pattern, which may be defined

MSC2010: primary 11E04; secondary 14E05, 15A03.

Keywords: quadratic forms, quasilinear $p$-forms, splitting patterns, canonical dimension. 
as the increasing sequence of nonzero isotropy indices attained by $\phi$ under scalar extension to every overfield of $k{ }^{1}$ Although this sequence appears to be somewhat intractable in general, its first entry (assuming there is one) may be computed more explicitly as the isotropy index of the extension $\phi_{L}$ of $\phi$ to the function field $L=k\left(X_{\phi}\right)$ of the (integral) quadric $X_{\phi}$. This (almost tautological) observation is the basic motivation underlying the following construction, originally due to Knebusch [1976]: let $k_{0}=k, \phi_{0}=\phi$, and inductively define $k_{r}=k_{r-1}\left(X_{\phi_{r-1}}\right)$, $\phi_{r}=\left(\phi_{k_{r}}\right)_{\mathrm{an}},{ }^{2}$ with the understanding that this (finite) process stops when we reach the first nonnegative integer $h(\phi)$ such that $\operatorname{dim} \phi_{h(\phi)} \leq 1$. The integer $h(\phi)$ and the tower of fields $k=k_{0} \subset k_{1} \subset \cdots \subset k_{h(\phi)}$ are known as the height and Knebusch splitting tower of $\phi$, respectively. For $1 \leq r \leq h(\phi)$, the anisotropic form $\phi_{r}$ is called the $r$-th higher anisotropic kernel of $\phi$. The $r$-th higher isotropy index of $\phi$, denoted $\mathfrak{i}_{r}(\phi)$, is defined as the difference $\mathfrak{i}_{0}\left(\phi_{k_{r}}\right)-\mathfrak{i}_{0}\left(\phi_{k_{r-1}}\right)$. The sequence $\mathfrak{i}(\phi)=\left(\mathfrak{i}_{1}(\phi), \ldots, \mathfrak{i}_{h(\phi)}(\phi)\right)$ is called the Knebusch splitting pattern of $\phi .{ }^{3}$ Note that we have $\mathfrak{i}_{r}(\phi)=\mathfrak{i}_{1}\left(\phi_{r-1}\right)$ for every $r \geq 2$ by the inductive nature of the construction.

If $\phi$ is nonsingular, then its full and Knebusch splitting patterns are easily seen to determine one another (see [Elman et al. 2008, Proposition 25.1]). This need not be the case for (totally) singular forms (see Example 2.47 below), but Knebusch's construction still offers a meaningful and practical way to preclassify quadratic forms according to some notion of "algebraic complexity". By virtue of its definition, the Knebusch splitting pattern thus embodies a fundamental link between intrinsic algebraic properties of quadratic forms and the geometry of the algebraic varieties which are naturally associated to them. In recent years, the advent of effective new tools with which to study algebraic cycles on projective homogeneous varieties has, in this way, led to dramatic progress on many long-standing problems within the algebraic theory of quadratic forms. The impact of these developments has been felt most deeply in characteristic $\neq 2$, where (1) anisotropic forms of dimension $\geq 2$ are necessarily nonsingular, and (2) the geometric methods are better developed, even if we restrict our considerations to nonsingular forms only; see [Elman et al. 2008] for a thorough exposition of much of the recent work which has been done in this area.

One of the central problems in the investigation of splitting properties of quadratic forms over general fields is the following:

\footnotetext{
${ }^{1}$ This terminology is not standard; see, e.g., [Hoffmann and Laghribi 2004], where refined splitting pattern invariants are considered. Our definition does, however, agree (in content if not presentation) with those found in the literature when $\phi$ is nonsingular or totally singular (the only relevant cases here).

${ }^{2}$ For any form $\psi$ over a field $K, \psi$ an denotes the anisotropic kernel of $\psi$, an anisotropic $K$-form uniquely determined up to isomorphism by the (refined) Witt decomposition of $\psi$; see [Hoffmann and Laghribi 2004, §2].

${ }^{3}$ The term standard splitting pattern is also used in the literature. Footnote 1 again applies here.
} 
Question 1.1. Let $\phi$ be an anisotropic quadratic form of dimension $\geq 2$ over a field. What are the possible values of the sequence $\mathfrak{i}(\phi)$ ?

Since $\mathfrak{i}_{r}(\phi)=\mathfrak{i}_{1}\left(\phi_{r-1}\right)$ for all $2 \leq r \leq h(\phi)$, the natural first approximation to this problem is to determine the possible values of the invariant $\mathfrak{i}_{1}$ among all forms of a given dimension. To this end, we have the following general conjecture:

Conjecture 1.2 (Hoffmann $\left.{ }^{4}\right)$. Let $\phi$ be an anisotropic quadratic form of dimension $\geq 2$ over a field. Then $\mathfrak{i}_{1}(\phi)-1$ is the remainder modulo $2^{s}$ of $\operatorname{dim} \phi-1$ for some $s<\log _{2}(\operatorname{dim} \phi){ }^{5}$

In characteristic $\neq 2$, the first major result in the direction of Conjecture 1.2 was established by Hoffmann himself [1995, Corollary 1], who showed that if $\operatorname{dim} \phi=2^{n}+m$ for nonnegative integers $n$ and $1 \leq m \leq 2^{n}$, then $\mathfrak{i}_{1}(\phi) \leq m$. A few years later, Izhboldin [2004, Corollary 5.12] proved that one cannot have $\mathfrak{i}_{1}(\phi)=m-1$ here unless $m=2$. Izhboldin's paper combined an elaboration of the algebraic methods conceived in [Hoffmann 1995] with emerging work of Vishik [1998; 1999], who developed a systematic approach to the study of the splitting pattern using the (integral) motive of the given quadric (see [Vishik 2004], where motivic methods were used to verify Hoffmann's conjecture in all dimensions $\leq 22$ in this setting). Going further, Vishik later formulated a very general conjecture concerning the complete motivic decomposition of a smooth anisotropic quadric (the excellent connections conjecture) which subsumed the nonsingular case of Conjecture 1.2 in a conceptual way. Not long after this, Karpenko [2003] used a similar approach to prove the characteristic $\neq 2$ case of the conjecture in its entirety, the key new ingredient being the use of (reduced power) Steenrod operations on modulo-2 Chow groups. More recently, Vishik [2011, Theorem 1.3] proved the excellent connections conjecture in characteristic $\neq 2$, thus yielding another proof of Hoffmann's conjecture in this setting. Vishik's work also makes essential use of Steenrod squares in Chow theory.

In characteristic 2, the general picture is more complicated. In the nonsingular case, Karpenko and Vishik's approaches to Conjecture 1.2 are still valid, and progress is only hindered here by the fact that the total mod-2 Steenrod operation is not yet available when 2 is not invertible in the base field. To this end, weak forms of the first three Steenrod squares have been constructed by Haution [2013, Theorem 6.2; 2015, Theorem 5.8], and these suffice to prove nontrivial partial

\footnotetext{
${ }^{4}$ This conjecture was originally stated by Hoffmann under the additional hypothesis $\operatorname{char}(k) \neq 2$, but we expect that the assertion is also valid in characteristic 2 .

${ }^{5}$ After passing to a purely transcendental extension of $k$ if necessary, all values of $\mathfrak{i}_{1}(\phi)$ which are not excluded by the conjecture can be realized by making an appropriate choice of $\phi$. In all cases, $\phi$ may, in fact, be chosen to be either nonsingular or (if $\operatorname{char}(k)=2$ ) totally singular; see [Vishik 2004, $\S 7.4]$ and Proposition 6.4 below, respectively.
} 
results towards Conjecture 1.2. Haution's results are further supplemented by earlier work of Hoffmann and Laghribi [2006, Lemma 4.1], who extended Hoffmann's upper bound on $\mathfrak{i}_{1}(\phi)$ to the characteristic-2 setting, irrespective of whether $\phi$ is nonsingular or not. For singular forms, however, the situation is different, and almost nothing is known in the direction of Conjecture 1.2 beyond Hoffmann and Laghribi's bound. In fact, the only real exception to this general state of affairs lies in the extreme case where $\phi$ is totally singular. Here, it was recently shown in [Scully 2016, Theorem 9.4] that if $\operatorname{dim} \phi=2^{n}+m$ for nonnegative integers $n$ and $1 \leq m \leq 2^{n}$, and if $\mathfrak{i}_{1}(\phi) \neq m$ (that is, if Hoffmann and Laghribi's bound is not met), then $\mathfrak{i}_{1}(\phi) \leq m / 2$. In the present article, we will settle this case completely by proving:

\section{Theorem 1.3. Conjecture 1.2 is true in the case where $\phi$ is totally singular.}

Contrary to the existing approaches to the nonsingular case of Conjecture 1.2, our proof of Theorem 1.3 does not involve the study of Chow correspondences on the quadric $X_{\phi}$. Indeed, although we also make use of the computation of the canonical dimension ${ }^{6}$ of $X_{\phi}$ (see [Karpenko and Merkurjev 2003; Totaro 2008]), it is exploited here in a rather more direct and algebraic way. This point of view begins with the following observation:

Proposition 1.4 (see Proposition 4.3 below). Let $\phi$ be an anisotropic totally singular quadratic form of dimension $\geq 2$ over a field $k$ of characteristic 2 and let $\psi \subset \phi$ be a subform of codimension $\mathfrak{i}_{1}(\phi)$. Suppose furthermore that $h(\psi)<h(\phi) .{ }^{7}$ Then there exist a quasi-Pfister quadratic form $\pi,{ }^{8}$ a subform $\sigma \subset \pi$, an element $\lambda \in k^{*}$ and a form $\tau$ over $k$ such that $\psi \simeq \pi \otimes \tau$ and $\phi \simeq \psi \perp \lambda \sigma$.

In the situation of Proposition 1.4, Hoffmann's conjecture is immediately verified. Indeed, (since $\sigma \subset \pi$ ) the integer $\operatorname{dim} \pi$ is a power of 2 strictly greater than $\mathfrak{i}_{1}(\phi)-1=\operatorname{dim} \sigma-1$, and (since $\psi$ is divisible by $\pi$ ) we have $\operatorname{dim} \phi-1=$ $\operatorname{dim} \psi+\mathfrak{i}_{1}(\phi)-1 \equiv \mathfrak{i}_{1}(\phi)-1(\bmod \operatorname{dim} \pi)$. It is not always possible, however, to decompose the form $\phi$ in the manner intimated by the proposition. In fact, Vishik (see [Totaro 2009, Lemma 7.1]) has given examples of 16-dimensional anisotropic quadratic forms in characteristic $\neq 2$ which have first higher isotropy index equal to 2, but which do not decompose in this way, and the same examples carry over into the totally singular setting (see Lemma 4.4 below). Thus, the picture is, in general, more complicated than that suggested by Proposition 1.4. Perhaps surprisingly, however, the main result of this paper shows that the next best thing happens:

\footnotetext{
${ }^{6}$ Here, the canonical dimension of an algebraic variety $X$ over a field $k$ should be understood as the minimal dimension $\operatorname{cdim}(X)$ of the image of a rational self-map $X \rightarrow X$.

${ }^{7}$ A weaker condition will suffice; see the statement of Proposition 4.3.

${ }^{8}$ That is, $\pi$ is the diagonal part of a bilinear Pfister form over $k$; see Section $2 \mathrm{C}$ below.
} 
Theorem 1.5. Let $\phi$ be an anisotropic totally singular quadratic form of dimension $\geq 2$ over a field of characteristic 2 and let s be the smallest nonnegative integer such that $2^{s} \geq \mathfrak{i}_{1}(\phi)$. Then $\phi_{1}$ is divisible by an $s$-fold quasi-Pfister form.

This is a new kind of statement of which no analogue is known in the nonsingular theory (even in characteristic $\neq 2$ ). As remarked above, a key ingredient needed for its proof is Totaro's computation [2008, Theorem 5.1] of the canonical dimension of a totally singular quadric. In [Scully 2013], this computation was extended to the wider class of Fermat-type hypersurfaces of degree $p$ over fields of characteristic $p>0$, and this enables us to also prove a direct analogue of Theorem 1.5 for totally singular forms of any prime degree $p>2$ (known here as quasilinear p-forms); see Theorem 5.1 below. Subsequently, we also get an analogue of Theorem 1.3 in higher degrees. As a corollary, this yields a complete solution to the problem of determining the possible values of the canonical dimension of a degree- $p$ Fermat-type hypersurface in characteristic $p>0$ (Theorem 6.6); it is worth noting here that no such result is known for Fermat-type hypersurfaces of prime degree $p>2$ over fields of characteristic not $p$. Returning to the case where $p=2$, let us explain more precisely how Theorem 1.5 implies the totally singular case of Hoffmann's conjecture:

Proof of Theorem 1.3. Since $\phi$ is totally singular, we have $\operatorname{dim} \phi_{1}=\operatorname{dim} \phi-\mathfrak{i}_{1}(\phi)$ (see Remarks 2.36(2) below). Thus, if $s$ is as in Theorem 1.5, then

$$
\operatorname{dim} \phi-1=\operatorname{dim} \phi_{1}+\mathfrak{i}_{1}(\phi)-1 \equiv \mathfrak{i}_{1}(\phi)-1\left(\bmod 2^{s}\right) .
$$

Since $\mathfrak{i}_{1}(\phi)-1<2^{s}$, the result follows.

Of course, our main result goes somewhat deeper than this. In fact, Theorem 1.5 (resp. Theorem 5.1 below) yields a complete answer to Question 1.1 in the totally singular case (resp. its analogue for arbitrary quasilinear $p$-forms). In other words, all restrictions on the possible values of the Knebusch splitting pattern of $\phi$ are explained here by the presence of certain divisibilities among its higher anisotropic kernels - precise statements are given in Section 6A below (see Theorem 6.1, Proposition 6.4). With this result in hand, it then becomes natural to try to understand the general discrepancy which exists between the Knebusch and full splitting patterns in the totally singular case. An immediate challenge here concerns the determination of all nontrivial restrictions which the former invariant imposes on the latter. In Section 7 below, we initiate this process by conjecturing that the gaps in the full splitting pattern established in characteristic $\neq 2$ by Vishik [2011, Proposition 2.6] (or see Theorem 7.1 below) as a consequence of his proof of the excellent connections conjecture are also present in the totally singular 
theory. A particular case of this conjecture was already proved in [Scully 2016, Theorem 9.2], and we provide some further evidence for its general veracity here. ${ }^{9}$

Finally, the main results of this paper should have valuable implications for the study of symmetric bilinear forms over fields of characteristic 2. Indeed, in characteristic 2, the diagonal parts of symmetric bilinear forms are nothing else but the totally singular quadratic forms discussed above. This will be investigated in a later text.

The remainder of this text is organized as follows. In Sections 2 and 3, we recall the basic theory of quasilinear $p$-forms and introduce the key notions and results which will be needed in the main part of the text. As a warm-up for the proof of our main result, we prove in Section 4 (a stronger version of) Proposition 1.4 and consider some situations in which it may be applied. The proof of Theorem 1.5 (and its generalization to higher degrees) is then given in Section 5, and, in Section 6, we apply this result to determine all possible Knebusch splitting patterns of quasilinear $p$-forms and settle another conjecture of Hoffmann concerning quasilinear $p$-forms with "maximal splitting". Lastly, in Section 7, we consider the aforementioned problem of establishing totally singular analogues of the results obtained in [Vishik 2011].

Notation and Terminology. Unless stated otherwise, $p$ will denote an arbitrary prime integer and $F$ will denote an arbitrary field of characteristic $p$. If $L$ is a field of characteristic $p$ and $a_{1}, \ldots, a_{n}$ are elements of $L$, then $L_{a_{1}, \ldots, a_{n}}$ will denote the field $L\left(\sqrt[p]{a_{1}}, \ldots, \sqrt[p]{a_{n}}\right)$. Finally, if $k$ is a field and $T=\left(T_{1}, \ldots, T_{m}\right)$ is a tuple of algebraically independent variables over $k$, then we will write $k[T]$ for the polynomial ring $k\left[T_{1}, \ldots, T_{n}\right]$ and $k(T)$ for its fraction field.

\section{Quasilinear $p$-forms and quasilinear $p$-hypersurfaces}

The basic material presented in this section was originally developed in the series of papers [Hoffmann and Laghribi 2004; Laghribi 2004a; 2004b; 2006; Hoffmann 2004]. Additional elementary results which will be needed in the sequel are also included here. For any details which are omitted from our exposition of the basic theory, we refer the reader to [Hoffmann 2004].

2A. Basic notions. Let $\phi: V \rightarrow F$ be an $F$-valued form on a finite-dimensional $F$-vector space $V$. We say that $\phi$ is a quasilinear $p$-form (on $V$ ) if $\phi$ is homogeneous of degree $p$ and the equation $\phi(v+w)=\phi(v)+\phi(w)$ holds for all $(v, w) \in V \times V$. By a quasilinear $p$-form over $F$ (or sometimes simply a form over $F$ or $F$-form), we will mean a quasilinear $p$-form on some finite-dimensional $F$-vector space. By a quasilinear p-hypersurface over $F$ we will mean a projective hypersurface defined by the vanishing of a nonzero quasilinear $p$-form on some $F$-vector space

\footnotetext{
${ }^{9}$ To the author's knowledge, there is no conjectural description of the possible values of the full splitting pattern, even in the nonsingular case (in any characteristic).
} 
of dimension $\geq 2$. In the special case where $p=2$, we will speak of quasilinear quadratic forms rather than quasilinear 2-forms.

Remark 2.1. Let $T=\left(T_{1}, \ldots, T_{n}\right)$ be a tuple of $n \geq 2$ algebraically independent variables over $F$, and let $f \in F[T] \backslash\{0\}$. Then the projective hypersurface $X_{f}=\{f=0\} \subset \mathbb{P}^{n-1}$ is nowhere smooth if and only if $f \in F\left[T_{1}^{p}, \ldots, T_{n}^{p}\right]$. In particular, if $p=2$, then a nonzero quadratic form of dimension $\geq 2$ over $F$ is totally singular (in the sense of Section 1) if and only if it is quasilinear.

Let $\phi$ be a quasilinear $p$-form over $F$. The underlying $F$-vector space of $\phi$ will be denoted by $V_{\phi}$. Its dimension will be called the dimension of $\phi$ and will be denoted by $\operatorname{dim} \phi$. If $\operatorname{dim} \phi \geq 2$ and $\phi$ is nonzero, then the quasilinear $p$-hypersurface $\{\phi=0\} \subset \mathbb{P}\left(V_{\phi}\right)$ (which is nowhere smooth by Remark 2.1) will be denoted by $X_{\phi}$. The set $\left\{\phi(v) \mid v \in V_{\phi}\right\}$ of elements of $F$ represented by $\phi$ will be denoted by $D(\phi)$. Given a field extension $L$ of $F$, we will write $\phi_{L}$ for the unique quasilinear $p$-form on the $L$-vector space $V_{\phi} \otimes_{F} L$ such that $\phi_{L}(v \otimes 1)=\phi(v)$ for all $v \in V_{\phi}$. If $R$ is a subring of $L$ containing $F$, then $D\left(\phi_{R}\right)$ will denote the subset $\left\{\phi(w) \mid w \in V_{\phi} \otimes_{F} R\right\}$ of $D\left(\phi_{L}\right)$ (which lies in $R$ ). Given $a \in F$, we will write $a \phi$ for the form $v \mapsto a \phi(v)$ on the vector space $V_{\phi}$.

Let $\psi$ be another quasilinear $p$-form over $F$. If there exists an injective (resp. bijective) F-linear map $f: V_{\psi} \rightarrow V_{\phi}$ such that $\phi(f(v))=\psi(v)$ for all $v \in V_{\psi}$, then we will say that $\psi$ is a subform of (resp. is isomorphic to) $\phi$ and write $\psi \subset \phi$ (resp. $\psi \simeq \phi$ ). If $\psi \simeq a \phi$ for some $a \in F^{*}$, then we will say that $\psi$ and $\phi$ are similar. The sum $\psi \oplus \phi$ (resp. product $\psi \otimes \phi$ ) is defined as the unique quasilinear $p$-form on $V_{\psi} \oplus V_{\phi}$ (resp. $\left.V_{\psi} \otimes_{F} V_{\phi}\right)$ such that $(\psi \oplus \phi)((v, w))=\psi(v)+\phi(w)$ (resp. $(\psi \otimes \phi)(v \otimes w)=\psi(v) \phi(w))$ for all $(v, w) \in V_{\psi} \times V_{\phi}$. Given a positive integer $n$, we will let $n \cdot \phi$ denote the sum of $n$ copies of $\phi$ (note that we have $n \cdot \phi \neq n \phi$ for $n>1$ ). If there exists a form $\tau$ over $F$ such that $\phi \simeq \psi \otimes \tau$, then we will say that $\phi$ is divisible by $\psi$.

Given elements $a_{1}, \ldots, a_{n} \in F$, we will write $\left\langle a_{1}, \ldots, a_{n}\right\rangle$ for the quasilinear $p$-form $\left(\lambda_{1}, \ldots, \lambda_{n}\right) \mapsto \sum_{i=1}^{n} a_{i} \lambda_{i}^{p}$ on the $F$-vector space $F^{\oplus n}$. By definition, every quasilinear $p$-form of dimension $n$ over $F$ is isomorphic to $\left\langle a_{1}, \ldots, a_{n}\right\rangle$ for some $a_{i} \in F$.

A vector $v \in V_{\phi}$ is said to be isotropic if $\phi(v)=0$. We will say that $\phi$ is isotropic if $V_{\phi}$ contains a nonzero isotropic vector, and anisotropic otherwise. By the additivity of $\phi$, the subset $V_{\phi}^{0}$ of all isotropic vectors in $V_{\phi}$ is, in fact, an $F$-linear subspace of $V_{\phi}$. Its dimension will be called the isotropy index of $\phi$, and will be denoted by $\mathfrak{i}_{0}(\phi)$ (note that in the case where $p=2$ and $\phi$ is nonzero, this agrees with the definition given in Section 1). The additivity of $\phi$ also implies that $D(\phi)$ is a finite-dimensional $F^{p}$-linear subspace of $F$ (where $F$ is equipped with its natural $F^{p}$-vector space structure). Conversely, if $U$ is a nonzero finite-dimensional 


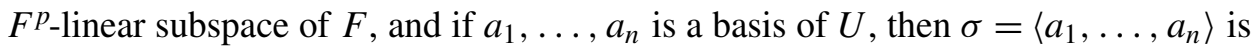
a quasilinear $p$-form over $F$ satisfying $D(\sigma)=U$. In fact, it is easy to see that, up to isomorphism, $\sigma$ is the unique anisotropic quasilinear $p$-form with this property:

Lemma 2.2 (see [Hoffmann 2004, Proposition 2.12]). Let $U$ be a finite-dimensional $F^{p}$-linear subspace of $F$. Then, up to isomorphism, there exists a unique anisotropic quasilinear $p$-form $\phi$ over $F$ such that $D(\phi)=U$.

In particular, Pfister's quadratic subform theorem [Elman et al. 2008, Theorem 17.12] takes the following simplified form in this setting:

Proposition 2.3 (see [Hoffmann 2004, Proposition 2.6]). Let $\psi$ and $\phi$ be anisotropic quasilinear $p$-forms over $F$. Then $\psi \subset \phi$ if and only if $D(\psi) \subseteq D(\phi)$. In particular, $\psi \simeq \phi$ if and only if $D(\psi)=D(\phi)$.

In view of these observations, we can define (up to isomorphism) the anisotropic kernel of $\phi$ as the unique anisotropic quasilinear $p$-form $\phi_{\text {an }}$ over $F$ such that $D\left(\phi_{\mathrm{an}}\right)=D(\phi)$. If we view $D(\phi)$ as an $F$-vector space via the Frobenius $F \mapsto F^{p}$, then $\phi: V_{\phi} \rightarrow D(\phi)$ is a surjective $F$-linear map with kernel $V_{\phi}^{0}$. We thus obtain:

Proposition 2.4 (see [Hoffmann 2004, Lemma 2.10]). Let $\phi$ be a quasilinear $p$-form over $F$. Then $\phi$ is anisotropic if and only if $\phi \simeq \phi_{\mathrm{an}}$. If $\phi$ is isotropic, then $\phi \simeq \phi_{\mathrm{an}} \oplus \mathfrak{i}_{0}(\phi) \cdot\langle 0\rangle$. In particular, $\operatorname{dim} \phi_{\mathrm{an}}=\operatorname{dim} \phi-\mathfrak{i}_{0}(\phi)$.

In summary, we see that $\phi$ is determined up to isomorphism by the set $D(\phi)$ and the integer $\mathfrak{i}_{0}(\phi)$. If $\operatorname{dim} \phi_{\text {an }} \leq 1$, then we will say that $\phi$ is split. Given another form $\psi$ over $F$, we will write $\psi \sim \phi$ whenever $\psi_{\text {an }} \simeq \phi_{\text {an }}$. If $F$ is perfect (that is, if $F=F^{p}$ ), then every form over $F$ is split and the theory is vacuous. As such, we are essentially only interested in the case where $F$ is imperfect. Unless indicated otherwise, we will assume henceforth that all quasilinear $p$-forms are nonzero (i.e., of anisotropic dimension $\geq 1$ ).

Remark 2.5. Let $\phi$ be a quasilinear $p$-form over $F$. Choose a basis $v_{1}, \ldots, v_{n}$ of $V_{\phi}$ and let $a_{i}=\phi\left(v_{i}\right)$ for all $1 \leq i \leq n$, so that $\phi \simeq\left\langle a_{1}, \ldots, a_{n}\right\rangle$. Suppose that $v \in V_{\phi}$ is an isotropic vector, and write $v=\sum_{i=1}^{n} \lambda_{i} v_{i}$. If $\lambda_{j} \neq 0$ for $1 \leq j \leq n$, then the $F^{p}$-vector space $D(\phi)$ is spanned by the elements $a_{1}, \ldots, a_{j-1}, a_{j+1}, \ldots, a_{n}$. In particular, we have $\phi \sim\left\langle a_{1}, \ldots, a_{j-1}, a_{j+1}, \ldots, a_{n}\right\rangle$.

2B. Function fields of quasilinear p-hypersurfaces and their products. Let $\phi$ be a quasilinear $p$-form of dimension $\geq 2$ over $F$. If $\phi$ is not split, then the quasilinear $p$-hypersurface $X_{\phi}$ is an integral scheme (see [Hoffmann 2004, Lemma 7.1]), as is its affine cone $\{\phi=0\} \subset \mathbb{A}\left(V_{\phi}\right)$. In this case, we will write $F(\phi)$ for the function field of the former and $F[\phi]$ for that of the latter. If $L$ is a field extension of $F$, then we will simply write $L(\phi)$ instead of $L\left(\phi_{L}\right)$ whenever it is defined. In general, given a finite collection $\phi_{1}, \ldots, \phi_{n}$ of quasilinear $p$-forms of dimension $\geq 2$ over $F$, 
we will write $F\left(\phi_{1} \times \cdots \times \phi_{n}\right)$ for the function field of the scheme $X_{\phi_{1}} \times \cdots \times X_{\phi_{n}}$, provided that it is integral. This notation will be further simplified where possible; for example, if $\phi_{1}=\cdots=\phi_{n}=\phi$, then we will simply write $F\left(\phi^{\times n}\right)$ instead of $F\left(\phi_{1} \times \cdots \times \phi_{n}\right)$.

Remarks 2.6. Let $\phi$ be a quasilinear $p$-form over $F$. Assume that $\phi$ is not split. We make the following basic observations:

(1) Let $a_{0}, \ldots, a_{n} \in F$ be such that $\phi \simeq\left\langle a_{0}, \ldots, a_{n}\right\rangle$ and $a_{0}, a_{1} \neq 0$. Then we have $F$-isomorphisms

$$
F(\phi) \simeq F(S)\left(\sqrt[p]{a_{1}^{-1}\left(a_{0}+a_{2} S_{2}^{p}+\cdots+a_{n} S_{n}^{p}\right)}\right)
$$

and

$F[\phi] \simeq \operatorname{Frac}\left(F[T] /\left(a_{0} T_{0}^{p}+\cdots+a_{n} T_{n}^{p}\right)\right) \simeq F(U)\left(\sqrt[p]{a_{0}^{-1}\left(a_{1} U_{1}^{p}+\cdots+a_{n} U_{n}^{p}\right)}\right)$, where $S=\left(S_{2}, \ldots, S_{n}\right), T=\left(T_{0}, \ldots, T_{n}\right)$ and $U=\left(U_{1}, \ldots, U_{n}\right)$ are tuples of algebraically independent variables over $F$.

(2) $F[\phi]$ is $F$-isomorphic to a degree-1 purely transcendental extension of $F(\phi)$.

(3) $F(\phi)$ is $F$-isomorphic to a degree-i $i_{0}(\phi)$ purely transcendental extension of $F\left(\phi_{\text {an }}\right)$; see Proposition 2.4 .

(4) The form $\phi_{F(\phi)}$ is evidently isotropic. Furthermore, if $a_{1}, \ldots, a_{n} \in F$ are such that $\phi \simeq\left\langle a_{1}, \ldots, a_{n}\right\rangle$, then consideration of the generic point in $X_{\phi}(F(\phi))$ shows that $\phi_{F(\phi)} \sim\left\langle a_{1}, \ldots, a_{i-1}, a_{i+1}, \ldots, a_{n}\right\rangle$ for every $1 \leq i \leq n$; see Remark 2.5.

2C. Quasi-Pfister p-forms. Let $\phi$ be a quasilinear $p$-form over $F$ and let $n$ be a positive integer. We say that $\phi$ is an $n$-fold quasi-Pfister $p$-form if there exist $a_{1}, \ldots, a_{n} \in F$ such that $\phi \simeq\left\langle\left\langle a_{1}, \ldots, a_{n}\right\rangle\right\rangle:=\bigotimes_{i=1}^{n}\left\langle 1, a_{i}, a_{i}^{2}, \ldots, a_{i}^{p-1}\right\rangle$. For convenience, we also say that $\phi$ is a 0 -fold quasi-Pfister $p$-form if $\phi \simeq\langle 1\rangle$. Note, in particular, that if $\phi$ is an $n$-fold quasi-Pfister $p$-form for some $n \geq 0$, then we have $\operatorname{dim} \phi=p^{n}$. The basic observation concerning quasi-Pfister $p$-forms is found in the following proposition (which follows easily from Lemma 2.2):

Proposition 2.7 (see [Hoffmann 2004, Proposition 4.6]). Let $\phi$ be a quasilinear $p$-form over $F$. Then $\phi_{\mathrm{an}}$ is a quasi-Pfister $p$-form if and only if $D(\phi)$ is a subfield of $F$.

Since the set of elements represented by an arbitrary quasi-Pfister $p$-form is, by definition, a subfield of the base field, we obtain:

Corollary 2.8 (see [Hoffmann 2004, Proposition 4.6]). Let $\phi$ be a quasi-Pfister p-form over $F$. Then $\phi_{\mathrm{an}}$ is a quasi-Pfister $p$-form. In particular, if $\phi$ is isotropic, then $\operatorname{dim} \phi_{\mathrm{an}}=\frac{1}{p^{k}} \operatorname{dim} \phi$ for some $k \geq 1$. 
Remark 2.9. More explicitly, let $\phi=\left\langle\left\langle a_{1}, \ldots, a_{n}\right\rangle\right\rangle$ for some $n \geq 1$ and $a_{i} \in F$. Then $D(\phi)=F^{p}\left(a_{1}, \ldots, a_{n}\right)$. Let $m$ be such that $\left[F^{p}\left(a_{1}, \ldots, a_{n}\right): F^{p}\right]=p^{m}$. If $m=0$ (i.e., $\left.D(\phi)=F^{p}\right)$, then $\phi_{\text {an }} \simeq\langle 1\rangle$. If $m \geq 1$, then $\phi_{\text {an }} \simeq\left\langle\left\langle b_{1}, \ldots, b_{m}\right\rangle\right\rangle$ for any $m$ elements $b_{1}, \ldots, b_{m} \in F$ such that $F^{p}\left(b_{1}, \ldots, b_{m}\right)=F^{p}\left(a_{1}, \ldots, a_{n}\right)$.

Quasi-Pfister $p$-forms have a central role to play in the general theory of quasilinear $p$-forms. As shown by Hoffmann [2004], these forms are distinguished here by the very same properties which distinguish the classical Pfister forms among nonsingular quadratic forms. For this reason, it will be useful to define the divisibility index of a given form $\phi$, denoted $\mathfrak{d}_{0}(\phi)$, as the largest nonnegative integer $s$ such that $\phi_{\text {an }}$ is divisible by an $s$-fold quasi-Pfister $p$-form. Clearly we have $\mathfrak{d}_{0}(\phi) \leq \log _{p}\left(\operatorname{dim} \phi_{\text {an }}\right)$, with equality holding if and only if $\phi_{\text {an }}$ is similar to a quasi-Pfister $p$-form. An alternative description of this invariant will be given in Corollary 2.19 below.

2D. The norm form. Let $\phi$ be a quasilinear $p$-form over $F$. The norm field of $\phi$, denoted $N(\phi)$, is defined (see [Hoffmann 2004, Definition 4.1]) as the smallest subfield of $F$ which contains all ratios of nonzero elements of $D(\phi)$. Note, in particular, that we have $N(a \phi)=N(\phi)=N\left(\phi_{\text {an }}\right)$ for all $a \in F^{*}$. In spite of its simple nature, this invariant has an important role to play in the whole theory. A more explicit description of the norm field may be given as follows:

Remark 2.10. If $a_{1}, \ldots, a_{n} \in F$ are such that $\phi \simeq\left\langle a_{1}, \ldots, a_{n}\right\rangle$ and $a_{1} \neq 0$, then we have $N(\phi)=F^{p}\left(\frac{a_{2}}{a_{1}}, \ldots, \frac{a_{n}}{a_{1}}\right)$.

In particular, we see that $N(\phi)$ is a nonzero finite-dimensional $F^{p}$-linear subspace of $F$. By Lemma 2.2, it follows that, up to isomorphism, there exists a unique anisotropic quasilinear $p$-form $\phi_{\text {nor }}$ over $F$ such that $D\left(\phi_{\text {nor }}\right)=N(\phi)$. The form $\phi_{\text {nor }}$ is called the norm form of $\phi$ (see [Hoffmann 2004, Definition 4.9]). By Proposition $2.7, \phi_{\text {nor }}$ is a quasi-Pfister $p$-form. Its dimension (which is necessarily equal to a power of $p$ ) is called the norm degree of $\phi$, and is denoted by ndeg $(\phi)$ (see [Hoffmann 2004, Definition 4.1]). The following lemma characterizes the norm form as the smallest anisotropic quasi-Pfister $p$-form which contains $\phi_{\text {an }}$ as a subform up to multiplication by a scalar (again, this is a simple consequence of Proposition 2.3):

Lemma 2.11 (see [Scully 2016, Lemma 2.10]). Let $\phi$ be a quasilinear p-form (resp. a quasilinear p-form such that $1 \in D(\phi)$ ) and $\pi$ an anisotropic quasi-Pfister $p$-form over $F$. Then $\phi_{\mathrm{an}}$ is similar to a subform of $\pi$ (resp. $\phi_{\mathrm{an}} \subset \pi$ ) if and only if $\phi_{\text {nor }} \subset \pi$. In particular, $\phi_{\text {an }}$ is similar to a subform of $\phi_{\text {nor }}$ (resp. $\phi_{\text {an }} \subset \phi_{\text {nor }}$ ).

Example 2.12. Let $\phi$ be a quasilinear $p$-form over $F$. The following are equivalent:

(1) $\phi_{\text {an }}$ is similar (resp. isomorphic) to a quasi-Pfister $p$-form. 
(2) $\phi_{\text {nor }} \simeq a \phi_{\text {an }}$ for some $a \in F^{*}$ (resp. $\left.\phi_{\text {nor }} \simeq \phi_{\text {an }}\right)$.

(3) $N(\phi)=a D(\phi)$ for some $a \in F^{*}($ resp. $N(\phi)=D(\phi))$.

2E. Similarity factors. Let $\phi$ be a quasilinear $p$-form over $F$. By a similarity factor of $\phi$, we mean an element $a \in F^{*}$ such that $a \phi \simeq \phi$. The set of all similarity factors of $\phi$ will be denoted by $G(\phi)^{*}$, and we will write $G(\phi)$ for the set $G(\phi)^{*} \cup\{0\}$. Note that $G(a \phi)=G(\phi)=G\left(\phi_{\text {an }}\right)$ for all $a \in F^{*}$, the second equality being an obvious consequence of Proposition 2.4. Thus, in view of Proposition 2.3, we have:

Lemma 2.13 (see [Hoffmann 2004, Lemma 6.3]). Let $\phi$ be a quasilinear p-form over $F$ and let $a \in F^{*}$. Then $a \in G(\phi)^{*}$ if and only if $a D(\phi) \subseteq D(\phi)$.

Example 2.14. Let $\phi$ be a quasi-Pfister $p$-form over $F$. Then, since $D(\phi)$ is a subfield of $F$, we have $G(\phi)=D(\phi)$.

More generally, Lemma 2.13 immediately implies the following:

Corollary 2.15 (see [Hoffmann 2004, Proposition 6.4]). Let $\phi$ be a quasilinear p-form over $F$. Then $G(\phi)$ is a subfield of $N(\phi)$ containing $F^{p}$.

In particular, $G(\phi)$ is a nonzero finite-dimensional $F^{p}$-linear subspace of $F$. By Lemma 2.2, it follows that, up to isomorphism, there exists a unique anisotropic quasilinear $p$-form $\phi_{\text {sim }}$ over $F$ such that $D\left(\phi_{\text {sim }}\right)=G(\phi)$. The form $\phi_{\text {sim }}$ is called the similarity form of $\phi$ (see [Hoffmann 2004, Definition 6.5]). By Proposition 2.7, $\phi_{\text {sim }}$ is a quasi-Pfister $p$-form. Taken together, Examples 2.12 and 2.14 yield:

Example 2.16. Let $\phi$ be a quasilinear $p$-form over $F$. The following are equivalent:

(1) $\phi_{\text {an }}$ is similar (resp. isomorphic) to a quasi-Pfister $p$-form.

(2) $\phi_{\text {sim }} \simeq \phi_{\text {nor }} \simeq a \phi_{\text {an }}$ for some $a \in F^{*}$ (resp. $\left.\phi_{\text {sim }} \simeq \phi_{\text {nor }} \simeq \phi_{\text {an }}\right)$.

(3) $G(\phi)=N(\phi)=a D(\phi)$ for some $a \in F^{*}(\operatorname{resp} . G(\phi)=N(\phi)=D(\phi))$.

The basic observation concerning similarity factors is the following:

Proposition 2.17. Let $\phi$ and $\psi$ be quasilinear $p$-forms over $F$. Then $G(\psi) \subseteq G(\phi)$ if and only if $\phi_{\mathrm{an}}$ is divisible by $\psi_{\mathrm{sim}}$.

Proof. We may assume that $1 \in D(\phi)$. Suppose $G(\psi) \subseteq G(\phi)$. By Corollary 2.15, $G(\psi)$ and $G(\phi)$ are subfields of $F$. By Lemma 2.13, $D(\phi)$ is naturally a (finitedimensional) vector space over $G(\phi)$, and hence over $G(\psi)$. If $a_{1}, \ldots, a_{m}$ is a basis of $D(\phi)$ over $G(\psi)$, then (since $D\left(\psi_{\text {sim }}\right)=G(\psi)$ ), Lemma 2.2 implies that $\phi_{\mathrm{an}} \simeq \psi_{\mathrm{sim}} \otimes\left\langle a_{1}, \ldots, a_{m}\right\rangle$. Conversely, if $\phi_{\mathrm{an}}$ is divisible by $\psi_{\mathrm{sim}}$, then it is clear that $G(\psi) \subseteq G(\phi)$, since $G(\psi)=D\left(\psi_{\text {sim }}\right)=G\left(\psi_{\text {sim }}\right)$ by Example 2.16.

We thus obtain the following characterization of the similarity form: 
Corollary 2.18 (see [Hoffmann 2004, Proposition 6.4]). Let $\phi$ be a quasilinear $p$-form and $\pi$ an anisotropic quasi-Pfister p-form over $F$. Then $\phi_{\mathrm{an}}$ is divisible by $\pi$ if and only if $\phi_{\mathrm{sim}}$ is divisible by $\pi$. In particular, $\phi_{\mathrm{an}}$ is divisible by $\phi_{\mathrm{sim}}$.

This enables us to reinterpret the divisibility index $\mathfrak{d}_{0}(\phi)$ (see Section 2C) as follows:

Corollary 2.19. Let $\phi$ be a quasilinear $p$-form over $F$. Then we have $\mathfrak{d}_{0}(\phi)=$ $\log _{p}\left(\operatorname{dim} \phi_{\text {sim }}\right)=\log _{p}\left(\left[G(\phi): F^{p}\right]\right)$.

We also get the following:

Corollary 2.20. Let $\phi$ and $\psi$ be quasilinear $p$-forms over $F$. Then $\phi_{\text {an }}$ is divisible by $\psi_{\text {nor }}$ if and only if $N(\psi) \subseteq G(\phi)$. If, additionally, $1 \in D(\psi)$, then the latter condition may be replaced by $D(\psi) \subseteq G(\phi)$.

Proof. For the second statement, we simply recall that $N(\phi)$ is the smallest subfield of $F$ containing all ratios of nonzero elements of $D(\phi)$ and that $G(\phi)$ is a subfield of $F$ (Corollary 2.15). For the first, we can replace $\psi$ by its norm form to arrive at the case where $N(\psi)=G(\psi)$ and $\psi_{\text {nor }} \simeq \psi_{\text {sim }}$ (see Example 2.16). The result is therefore a particular case of Proposition 2.17.

2F. A criterion for a quasilinear p-form to be quasi-Pfister. Let $\phi$ be a quasilinear $p$-form over $F$ such that $1 \in D(\phi)$, let $L$ be a field extension of $F$ and let $\alpha \in D\left(\phi_{L}\right) \backslash\{0\}$. Consider the set $S_{\alpha}=\left\{a \in F \mid \alpha a \in D\left(\phi_{L}\right)\right\}$. Since $D\left(\phi_{L}\right)$ is an $L^{p}$-linear subspace of $L$, we have the following observation:

$$
\sum \lambda_{i}^{p} a_{i} \in S_{\alpha} \quad \text { for all } \lambda_{i} \in F \text { and all } a_{i} \in S_{\alpha} .
$$

Lemma 2.21. In the above situation, let $P \in F^{p}[T]$ be a polynomial of degree $<p$ in a single variable $T$ such that $P(b) \in S_{\alpha}$ for all $b \in D(\phi)$. Then $b^{n} \in S_{\alpha}$ for all $b \in D(\phi)$ and all $n \leq \operatorname{deg}(P)$.

Proof. We proceed by induction on $d=\operatorname{deg}(P)$. Since $\alpha \in D\left(\phi_{L}\right)$, the case where $d=0$ is trivial. Suppose now that $d>0$, and let $\lambda \in F$ be such that $P\left(T+\lambda^{p}\right)=P(T)+Q(T)$ for some $Q \in F^{p}[T]$ of degree $d-1$. Since $F^{p} \subseteq D(\phi)$ by hypothesis, our assumption and (2-1) imply that $Q(b)=P\left(b+\lambda^{p}\right)-P(b) \in S_{\alpha}$ for all $b \in D(\phi)$. By the induction hypothesis, it follows that $b^{n} \in S_{\alpha}$ for all $b \in D(\phi)$ and all $n<d$. Finally, since $P(b)=\sum_{i=0}^{d} \lambda_{i}^{p} b^{i}$ for some $\lambda_{i} \in F$ with $\lambda_{d} \neq 0,(2-1)$ implies that, for any $b \in D(\phi)$, we also have $b^{d} \in S_{\alpha}$. This proves the lemma.

Suppose now that there exists a polynomial $P \in F^{p}[T]$ as in the statement of Lemma 2.21 with $\operatorname{deg}(P) \geq 2$ (in particular, we necessarily have $p>2$ ). A first application of the lemma shows that we have $D(\phi) \subseteq S_{\alpha}$. Since $D\left(\phi_{L}\right)$ is spanned by $D(\phi)$ as an $L^{p}$-vector space, this implies that $\alpha D\left(\phi_{L}\right) \subseteq D\left(\phi_{L}\right)$, and hence (for dimension reasons) that $\alpha D\left(\phi_{L}\right)=D\left(\phi_{L}\right)$. Another application of Lemma 2.21 
then shows that $b^{n} \in D\left(\phi_{L}\right)$ for all $b \in D(\phi)$ and all $n \leq \operatorname{deg}(P)$. In particular, since $\operatorname{deg}(P) \geq 2$, we have $2 b c=(b+c)^{2}-b^{2}-c^{2} \in D\left(\phi_{L}\right)$ for all $b, c \in D(\phi)$. Since $p>2$, and since $D\left(\phi_{L}\right)$ is spanned by $D(\phi)$ as an $L^{p}$-vector space, this implies that $D\left(\phi_{L}\right)$ is closed under multiplication, i.e., that $D\left(\phi_{L}\right)=N\left(\phi_{L}\right)$ (see Remark 2.10). By Example 2.12, this means that $\left(\phi_{L}\right)_{\text {an }} \simeq\left(\phi_{L}\right)_{\text {nor. }}$. We have thus proved:

Lemma 2.22. Assume that $p>2$. Let $\phi$ be a quasilinear $p$-form over $F$ such that $1 \in D(\phi)$ and let $L$ be a field extension of $F$. Suppose that there exists a polynomial $P \in F^{p}[T]$ in a single variable $T$, and an element $\alpha \in D\left(\phi_{L}\right) \backslash\{0\}$ such that $2 \leq \operatorname{deg}(P)<p$ and $\alpha P(b) \in D\left(\phi_{L}\right)$ for all $b \in D(\phi)$. Then $\left(\phi_{L}\right)_{\text {an }}$ is a quasi-Pfister p-form.

2G. The Cassels-Pfister representation theorem. Let $\phi$ be a quadratic form over a field $k$ and let $f \in k[T]$ be a polynomial in a single variable $T$ which is represented by the form $\phi_{k(T)}$. One of the foundational results of the classical algebraic theory of quadratic forms is the Cassels-Pfister representation theorem, which asserts that, in this case, $\phi$ already represents $f$ over the polynomial ring $k[T]$ (see [Elman et al. 2008, Theorem 17.3]). In the present setting, the original argument of Cassels may be readily adapted to prove the analogous statement for quasilinear $p$-forms. However, as pointed out by Hoffmann [2004], the additivity property of these forms enables one to prove a stronger multivariable statement taking the following form:

Theorem 2.23 (see [Hoffmann 2004, Corollary 3.4]). Let $\phi$ be a quasilinear p-form over $F$, let $T=\left(T_{1}, \ldots, T_{m}\right)$ be a tuple of algebraically independent variables over $F$ and let $f \in F[T]$. Then $f \in D\left(\phi_{F(T)}\right)$ if and only if $f \in D\left(\phi_{F[T]}\right)$, if and only if $f \in D(\phi)\left[T_{1}^{p}, \ldots, T_{m}^{p}\right]$.

Now, in the situation of Theorem 2.23, the $F(T)^{p}$-vector space $D\left(\phi_{F(T)}\right)$ is (evidently) spanned by elements of $D(\phi)$. Thus, in view of Lemma 2.13, we immediately obtain the following result concerning rational similarity factors:

Corollary 2.24 (see [Hoffmann 2004, Proposition 6.7]). Let $\phi$ be a quasilinear pform over $F$, let $T=\left(T_{1}, \ldots, T_{m}\right)$ be a tuple of algebraically independent variables over $F$ and let $f \in F[T]$. Then $f \in G\left(\phi_{F(T)}\right)$ if and only if $f \in G(\phi)\left[T_{1}^{p}, \ldots, T_{m}^{p}\right]$.

2H. Isotropy of quasilinear p-forms under scalar extension. We now collect some basic facts regarding the isotropy of quasilinear $p$-forms under scalar extension.

Let $K$ and $L$ be extensions of a field $k$. Recall that a $k$-place $K \rightarrow L$ is a pair $(R, f)$ consisting of a valuation subring $k \subseteq R \subseteq K$ and a local $k$-algebra homomorphism $f: R \rightarrow L$. For example, given an inclusion $i: K \hookrightarrow L$, the pair $(K, i)$ defines a $k$-place $K \rightarrow L$. If there exist $k$-places $K \rightarrow L$ and $L \rightarrow K$, then we say that $K$ and $L$ are equivalent over $k$, and write $K \sim_{k} L$. For instance, this is easily seen to be the case whenever $L$ (resp. $K$ ) is a purely transcendental 
extension of $K$ (resp. $L$ ) (see [Elman et al. 2008, §103] for further details). We have here the following basic lemma, which is a consequence of the completeness of $X_{\phi}$ (see [EGA II 1961, (7.3.8)]):

Lemma 2.25 (see [Scully 2016, Lemma 3.4]). Let $\phi$ be a quasilinear p-form over $F$ and let $K$ and $L$ be field extensions of $F$ such that there exists an $F$-place $K \rightarrow L$. Then $\mathfrak{i}_{0}\left(\phi_{L}\right) \geq \mathfrak{i}_{0}\left(\phi_{K}\right)$. In particular, if $K \sim_{F} L$, then $\mathfrak{i}_{0}\left(\phi_{K}\right)=\mathfrak{i}_{0}\left(\phi_{L}\right)$.

Note, in particular, that passage to rational extensions of the base field does not affect the isotropy index of a quasilinear $p$-form. By MacLane's theorem [Lang 2002, Proposition VIII.4], the same is, in fact, true of arbitrary separable extensions: ${ }^{10}$

Lemma 2.26 (see [Hoffmann 2004, Proposition 5.3]). Let $\phi$ be an anisotropic quasilinear $p$-form over $F$ and let $L$ be a field extension of $F$. If $L$ is separable over $F$, then $\phi_{L}$ is anisotropic and $\operatorname{ndeg}\left(\phi_{L}\right)=\operatorname{ndeg}(\phi)$.

Thus, in order to study the isotropy behavior of quasilinear $p$-forms under scalar extension, we are effectively reduced to considering the case of purely inseparable algebraic extensions. In degree $p$, we have the following basic observations, all of which can be easily verified using the results which have been discussed thus far (recall here that, given $a_{1}, \ldots, a_{n} \in F$, we denote by $F_{a_{1}, \ldots, a_{n}}$ the field $\left.F\left(\sqrt[p]{a_{1}}, \ldots, \sqrt[p]{a_{n}}\right)\right)$ :

Lemma 2.27 (see [Hoffmann 2004, §5; Scully 2016, Lemma 3.8]). Let $\phi$ be a quasilinear $p$-form over $F$ and let $a \in F \backslash F^{p}$. Then:

(1) $D\left(\phi_{F_{a}}\right)=D(\langle\langle a\rangle\rangle \otimes \phi)=\sum_{i=0}^{p-1} a^{i} D(\phi)$.

(2) $\mathfrak{i}_{0}\left(\phi_{F_{a}}\right)=\frac{1}{p} \mathfrak{i}_{0}(\langle\langle a\rangle\rangle \otimes \phi)$.

(3) $\operatorname{ndeg}\left(\phi_{F_{a}}\right)= \begin{cases}\frac{1}{p} \operatorname{ndeg}(\phi) & \text { if } a \in N(\phi) \text {, } \\ \operatorname{ndeg}(\phi) & \text { if } a \notin N(\phi) \text {. }\end{cases}$

(4) If $\phi$ is anisotropic and $a \notin N(\phi)$, then $\phi_{F_{a}}$ is anisotropic.

(5) $\operatorname{dim}\left(\phi_{F_{a}}\right)_{\mathrm{an}} \geq \frac{1}{p} \operatorname{dim} \phi_{\mathrm{an}}$.

(6) Equality holds in (5) if and only if $\phi_{\mathrm{an}}$ is divisible by $\langle\langle a\rangle$, if and only if $a \in G(\phi)$.

Remark 2.28. The second equivalence in (6) holds by Corollary 2.20.

As an application of the first part of the lemma, we have:

Corollary 2.29. Let $\phi$ be a quasilinear $p$-form over $F$ and let $a \in F \backslash F^{p}$. Then $G\left(\phi_{F_{a}}\right)=G(\langle\langle a\rangle\rangle \otimes \phi)$. In particular, $\mathfrak{d}_{0}(\langle\langle a\rangle\rangle \otimes \phi)=\mathfrak{d}_{0}\left(\phi_{F_{a}}\right)+1$.

${ }^{10}$ Recall that an extension of fields $k \subseteq L$ is called separable if, for any algebraic closure $\bar{k}$ of $k$, the ring $L \otimes_{k} \bar{k}$ has no nontrivial nilpotent elements. 
Proof. More specifically, the first statement is an immediate consequence of Lemmas 2.27(1) and 2.13. The second then follows from Corollary 2.19.

Suppose now that $\pi=\left\langle\left\langle a_{1}, \ldots, a_{n}\right\rangle\right\rangle$ is an anisotropic quasi-Pfister $p$-form over $F$. By Remark 2.9, we have $\left[F^{p}\left(a_{1}, \ldots, a_{n}\right): F^{p}\right]=p^{n}$, which means that $a_{i} \notin F_{a_{1}, \ldots, a_{i-1}}$ for every $1 \leq i \leq n$. Repeated applications of Lemma 2.27(2) and Corollary 2.29 therefore yield the following proposition:

Proposition 2.30. Let $\phi$ be a quasilinear $p$-form over $F$, let $\pi$ be as above and let $\psi=\pi \otimes \phi$. Then $\mathfrak{i}_{0}(\psi)=p^{n} \mathfrak{i}_{0}\left(\phi_{F_{a_{1}, \ldots, a_{n}}}\right)$ and $\mathfrak{d}_{0}(\psi)=\mathfrak{d}_{0}\left(\phi_{F_{a_{1}, \ldots, a_{n}}}\right)+n$.

Now, in view of Remarks 2.6(1), one may combine the above results in order to study the isotropy behavior of quasilinear $p$-forms under scalar extension to function fields of quasilinear $p$-hypersurfaces. More specifically, let $\psi$ be a quasilinear $p$-form over $F$ which is not split, and let $a_{0}, \ldots, a_{n} \in F$ be such that $\psi \simeq\left\langle a_{0}, \ldots, a_{n}\right\rangle$, with $a_{0}, a_{1} \neq 0$. Then, by Remarks 2.6(1), we have an $F$-isomorphism of fields

$$
F(\psi) \simeq F(T)\left(\sqrt[p]{a_{1}^{-1}\left(a_{0}+a_{2} T_{2}^{p}+\cdots+a_{n} T_{n}^{p}\right)}\right),
$$

where $T=\left(T_{2}, \ldots, T_{n}\right)$ is an $(n-1)$-tuple of algebraically independent variables over $F$. Thus, putting Lemmas 2.26 and 2.27 and together, we obtain:

Lemma 2.31 (see [Hoffmann 2004, $\S \S 7.3,7.4]$ ). Let $\phi$ be an anisotropic quasilinear $p$-form over $F$, and let $\psi$ be as above. Then:

(1) $\operatorname{dim}\left(\phi_{F(\psi)}\right)_{\text {an }} \geq \frac{1}{p} \operatorname{dim} \phi$.

(2) Equality holds in (1) if and only if $a_{1}^{-1}\left(a_{0}+a_{2} T_{2}^{p}+\cdots+a_{n} T_{n}^{p}\right) \in G\left(\phi_{F(T)}\right)$.

(3) $\operatorname{ndeg}\left(\phi_{F(\psi)}\right) \geq \frac{1}{p} \operatorname{ndeg}(\phi)$.

(4) Equality holds in (3) if and only if $a_{1}^{-1}\left(a_{0}+a_{2} T_{2}^{p}+\cdots+a_{n} T_{n}^{p}\right) \in N\left(\phi_{F(T)}\right)$.

(5) The equivalent conditions of (4) are satisfied if $\phi_{F(\psi)}$ is isotropic.

As a basic application, we have:

Corollary 2.32 (see [Hoffmann 2004, §§7.3, 7.4]). Let $\phi$ and $\psi$ be quasilinear p-forms over $F$ such that $\phi$ is anisotropic and $\psi$ is not split. Then:

(1) $\operatorname{dim}\left(\phi_{F(\psi)}\right)_{\mathrm{an}} \geq \frac{1}{p} \operatorname{dim} \phi$, with equality holding if and only if $N(\psi) \subseteq G(\phi)$.

(2) If $\phi_{F(\psi)}$ is isotropic, then $N(\psi) \subseteq N(\phi)$. In particular, $\operatorname{ndeg}(\psi) \leq \operatorname{ndeg}(\phi)$.

Proof. We may assume that $\psi$ is as in Lemma 2.31. In this case, we have $N(\psi)=$ $F^{p}\left(\frac{a_{0}}{a_{1}}, \ldots, \frac{a_{n}}{a_{1}}\right)$ (see Remark 2.9), and so (1) follows from the first two parts of the former lemma and Corollary 2.24. Similarly, since $N\left(\phi_{L}\right)=D\left(\left(\phi_{\mathrm{nor}}\right)_{L}\right)$ for any field extension $L$ of $F$, (2) follows from Lemma 2.31(4,5) and Theorem 2.23. 
Finally, it will be useful to record in this section another basic application of the Cassels-Pfister theorem. To state it, let $T=\left(T_{1}, \ldots, T_{m}\right)$ be a tuple of algebraically independent variables over $F$, let $g \in F[T]$ be an irreducible polynomial and let $F[g]$ denote the field $\operatorname{Frac}(F[T] /(g))$ (i.e., the function field of the integral hypersurface $\{g=0\} \subset \mathbb{A}_{F}^{m}$ ). Given $f \in F[T]$, we write $\operatorname{mult}_{g}(f)$ for the multiplicity of $g$ in $f$, i.e., the largest nonnegative integer $s$ such that $f=g^{s} h$ for some $h \in F[T]{ }^{11}$

Proposition 2.33. In the above situation, let $\phi$ be a quasilinear $p$-form over $F$ and let $f \in F[T]$. Suppose that $f \in D\left(\phi_{F(T)}\right)$ and that $\phi_{F[g]}$ is anisotropic. Then $\operatorname{mult}_{g}(f) \equiv 0(\bmod p)$.

Proof. Let $s=\operatorname{mult}_{g}(f)$. After replacing $f$ by $f / g^{k p} \in D\left(\phi_{F(T)}\right)$ for a suitable integer $k \geq 0$, we may assume that $s<p$. Our goal is then to prove that $s=0$. To see this, note first that there exists a $v \in V_{\phi} \otimes_{F} F[T]$ such that $\phi_{F(T)}(v)=f$ by Theorem 2.23. If $s \neq 0$, then the image $\bar{v}$ of $v$ in $V_{\phi} \otimes_{F} F[g]$ is an isotropic vector for $\phi_{F[g]}$. By hypothesis, it follows that $\bar{v}=0$, which means that $v=g w$ for some $w \in V_{\phi} \otimes_{F} F[T]$. But this implies that $f=g^{p} \phi_{F(T)}(w)$, which contradicts the fact that $s<p$. We conclude that $s=0$, and so the proposition is proved.

2I. The divisibility index and scalar extension. Let $\phi$ be a quasilinear $p$-form over $F$. We make some brief remarks concerning the behavior of the divisibility index $\mathfrak{d}_{0}(\phi)$ (see Section 2C) under scalar extension.

Lemma 2.34. Let $\phi$ be a quasilinear $p$-form over $F$ and let $L$ be a field extension of $F$. If $\left(\phi_{\mathrm{sim}}\right)_{L}$ is anisotropic, then $\mathfrak{d}_{0}\left(\phi_{L}\right) \geq \mathfrak{d}_{0}(\phi)$.

Proof. As an $L^{p}$-vector space, $D\left(\left(\phi_{\text {sim }}\right)_{L}\right)$ is spanned by $D\left(\phi_{\text {sim }}\right)=G(\phi)$. Since we evidently have $G(\phi) \subseteq G\left(\phi_{L}\right)=D\left(\left(\phi_{L}\right)_{\text {sim }}\right)$, and since $\left(\phi_{\text {sim }}\right)_{L}$ is anisotropic by hypothesis, Proposition 2.3 implies that $\left(\phi_{\text {sim }}\right)_{L} \subset\left(\phi_{L}\right)_{\text {sim. }}$. The desired assertion now follows from Corollary 2.19.

In particular, this applies in the case where $L$ is a separable extension of $F$ (see Lemma 2.26). In the case where $L$ is purely transcendental over $F$, we can say more:

Lemma 2.35. Let $\phi$ be a quasilinear $p$-form over $F$ and let $L$ be a purely transcendental extension of $F$. Then $\left(\phi_{L}\right)_{\operatorname{sim}} \simeq\left(\phi_{\mathrm{sim}}\right)_{L}$ and $\mathfrak{d}_{0}\left(\phi_{L}\right)=\mathfrak{d}_{0}(\phi)$.

Proof. Continuing with the proof of Lemma 2.34, it is sufficient to show that in this case $G\left(\phi_{L}\right)$ is generated by $G(\phi)$ over $L^{p}$. If $L$ is finitely generated over $F$, then this follows from Corollary 2.24. On the other hand, the general case reduces easily to the finitely generated case in view of Lemma 2.13, so the lemma is proved.

${ }^{11}$ With the added convention that $\operatorname{mult}_{g}(0)=+\infty$. 
2J. The Knebusch splitting pattern. Let $\phi$ be a quasilinear $p$-form over $F$. Following the construction outlined in Section 1 (see also [Hoffmann 2004, §7.5]), set $F_{0}=F, \phi_{0}=\phi_{\text {an }}$, and recursively define

- $F_{r}=F_{r-1}\left(\phi_{r-1}\right)$ (provided $\phi_{r-1}$ is not split), and

- $\phi_{r}=\left(\phi_{F_{r}}\right)_{\text {an }}$ (provided $F_{r}$ is defined).

Note here that if $\phi_{r}$ is defined, then we have $\operatorname{dim} \phi_{r}<\operatorname{dim} \phi_{r-1}$ by Remarks 2.6(4). As such, the whole process is finite, terminating at the first nonnegative integer $h(\phi)$ for which $\operatorname{dim} \phi_{h(\phi)} \leq 1$. The integer $h(\phi)$ will be called the height of $\phi$, and the tower of fields $F_{0} \subset F_{1} \subset \cdots \subset F_{h(\phi)}$ will be called the Knebusch splitting tower of $\phi$. For each $0 \leq r \leq h(\phi)$, we set $\mathfrak{j}_{r}(\phi)=\mathfrak{i}_{0}\left(\phi_{F_{r}}\right)$. If $\phi$ is not split and $r \geq 1$, then the difference $\mathfrak{j}_{r}(\phi)-\mathfrak{j}_{r-1}(\phi)$ will be called the $r$-th higher isotropy index of $\phi$, and will be denoted by $\mathfrak{i}_{r}(\phi)$. In this case, the form $\phi_{r}$ will be called the $r$-th higher anisotropic kernel of $\phi$. Finally, the sequence $\mathfrak{i}(\phi)=\left(\mathfrak{i}_{1}(\phi), \ldots, \mathfrak{i}_{h(\phi)}(\phi)\right)$ (understood to be empty if $\phi$ is split) will be called the Knebusch splitting pattern of $\phi .{ }^{12}$

Remarks 2.36. Let $\phi$ be a quasilinear $p$-form over $F$.

(1) By the recursive nature of the above construction, we have $\mathfrak{i}_{r}(\phi)=\mathfrak{i}_{1}\left(\phi_{r-1}\right)$ for every $1 \leq r \leq h(\phi)$.

(2) By Proposition 2.4, we have $\mathfrak{i}_{r}(\phi)=\operatorname{dim} \phi_{r-1}-\operatorname{dim} \phi_{r}$ for all $1 \leq r \leq h(\phi)$.

(3) Let $L$ be a field extension of $F$. As already remarked in Section 1, it is not true in general that $\mathfrak{i}_{0}\left(\phi_{L}\right)=\mathfrak{j}_{r}(\phi)$ for some $0 \leq r \leq h(\phi)$; see Example 2.47 below.

Note that by Remarks 2.6(3) we have the following:

Lemma 2.37. Let $\phi$ be a quasilinear $p$-form form of dimension $\geq 2$ and let $\left(F_{r}\right)$ denote its Knebusch splitting tower. Then $F_{r} \sim_{F} F\left(\phi^{\times r}\right)$ for every $0 \leq r \leq h(\phi)$.

In light of Lemma 2.37, we therefore have:

Corollary 2.38. Let $\phi$ be a quasilinear $p$-form over $F$. Then, for every $0 \leq r \leq h(\phi)$, we have $\mathfrak{j}_{r}(\phi)=\mathfrak{i}_{0}\left(\phi_{F\left(\phi^{\times r}\right)}\right)$.

Given the results of Section $2 \mathrm{H}$, we are now in a position to prove the following characterization of anisotropic quasi-Pfister $p$-forms:

Proposition 2.39 (see [Hoffmann and Laghribi 2004, Theorem 8.11]). Let $\phi$ be an anisotropic quasilinear $p$-form of dimension $\geq 2$ over $F$. Then $\operatorname{dim} \phi_{1} \geq \frac{1}{p} \operatorname{dim} \phi$, and the following conditions are equivalent:

(1) $\operatorname{dim} \phi_{1}=\frac{1}{p} \operatorname{dim} \phi$.

(2) $\mathfrak{i}(\phi)=\left(p^{h(\phi)}-p^{h(\phi)-1}, p^{h(\phi)-1}-p^{h(\phi)-2}, \ldots, p^{2}-p, p-1\right)$.

${ }^{12}$ See Footnote 3 . We omit the term $\mathfrak{i}_{0}(\phi)$ from the sequence because we are ultimately interested in the case where $\phi$ is anisotropic (i.e., where $\mathfrak{i}_{0}(\phi)=0$ ). 
(3) $\phi$ is similar to a quasi-Pfister $p$-form.

Proof. The inequality $\operatorname{dim} \phi_{1} \geq \frac{1}{p} \operatorname{dim} \phi$ holds by Corollary 2.32. The same result shows that equality holds if and only if $N(\phi) \subseteq G(\phi)$. By Corollary 2.20, the latter condition holds if and only if $\phi$ is divisible by $\phi_{\text {nor }}$. In view of Lemma 2.11 , this proves the equivalence of (1) and (3), as well as the implication (2) $\Rightarrow$ (3). On the other hand, if $\phi$ is similar to a quasi-Pfister $p$-form of dimension $p^{n}$, then $\phi_{1}$ is similar to a quasi-Pfister $p$-form of dimension $p^{n-1}$ by Corollary 2.8 and (1). Since $\mathfrak{i}_{1}(\phi)=\operatorname{dim} \phi-\operatorname{dim} \phi_{1}$ (see Remarks 2.36(2)), an easy induction on $h(\phi)$ then shows that (3) implies (2).

Finally, a repeated application of Lemma 2.31(3-5) (with $\psi=\phi$ ) yields the following computation of the height $h(\phi)$ :

Corollary 2.40 (see [Hoffmann 2004, Theorem 7.25(ii)]). Let $\phi$ be a quasilinear p-form over $F$. Then $h(\phi)=\log _{p}(\operatorname{ndeg}(\phi))$.

Together with Corollary 2.32(2), this implies the following useful result:

Corollary 2.41 (see [Scully 2016, Proposition 4.12]). Let $\phi$ and $\psi$ be quasilinear p-forms over $F$ such that $\phi$ is anisotropic and $\psi$ is not split. If $\phi_{F(\psi)}$ is isotropic, then $h(\psi) \leq h(\phi)$.

2K. The quasi-Pfister height and higher divisibility indices. Let $\phi$ be a quasilinear $p$-form over $F$. As in [Scully 2016, §4.2], we define the quasi-Pfister height of $\phi$, denoted $h_{\mathrm{qp}}(\phi)$, to be the smallest nonnegative integer $l$ such that $\phi_{l}$ is similar to a quasi-Pfister $p$-form (this is well defined, since $\phi_{h(\phi)}$, being of dimension 1 , is similar to a 0 -fold quasi-Pfister $p$-form). We have:

Lemma 2.42. Let $\phi$ be a quasilinear $p$-form over $F$ and let $d=h(\phi)-h_{\mathrm{qp}}(\phi)$. Then $\mathfrak{i}(\phi)=\left(\mathfrak{i}_{1}(\phi), \ldots, \mathfrak{i}_{h_{\mathrm{qp}}(\phi)}(\phi), p^{d}-p^{d-1}, p^{d-1}-p^{d-2}, \ldots, p^{2}-p, p-1\right)$ and $\mathfrak{i}_{h_{\mathrm{qp}}(\phi)}(\phi)=\operatorname{dim}(\phi)-\mathfrak{j}_{h_{\mathrm{qp}}(\phi)-1}(\phi)-p^{d}<p^{d+1}-p^{d}$.

Proof. The first statement is an immediate consequence of Proposition 2.39. The point here is that $\phi_{h_{\mathrm{qp}}(\phi)}$ is similar to a quasi-Pfister $p$-form of dimension $p^{d}$. By Remarks 2.36(2), we therefore have $\mathfrak{i}_{h_{\mathrm{qp}}(\phi)}(\phi)=\operatorname{dim} \phi_{h_{\mathrm{qp}}(\phi)-1}-\operatorname{dim} \phi_{h_{\mathrm{qp}}(\phi)}=$ $\operatorname{dim}(\phi)-\mathfrak{j}_{h_{\mathrm{qp}}(\phi)-1}(\phi)-p^{d}$. Finally, since $\phi_{h_{\mathrm{qp}}(\phi)-1}$ is (by the definition of $\left.h_{\mathrm{qp}}(\phi)\right)$ not similar to a quasi-Pfister $p$-form, it must have dimension $<p^{d+1}$, again by Proposition 2.39. This proves the inequality in the second statement, and hence the lemma.

The Knebusch splitting pattern of a quasilinear $p$-form $\phi$ is therefore determined by $h(\phi), h_{\mathrm{qp}}(\phi)$ and the truncated sequence $\left(\mathfrak{i}_{1}(\phi), \ldots, \mathfrak{i}_{h_{\mathrm{qp}}(\phi)}(\phi)\right)$. With a view to studying the latter invariant, we now introduce new invariants of $\phi$ which will be of central interest in the sequel. More specifically, for each $1 \leq r \leq h(\phi)$, we define the $r$-th higher divisibility index of $\phi$, denoted $\mathfrak{d}_{r}(\phi)$, as the integer $\mathfrak{d}_{0}\left(\phi_{r}\right)$ (see 
Section 2C). In other words, $\mathfrak{d}_{r}(\phi)$ is the largest integer $s$ such that $\phi_{r}$ is divisible by an $s$-fold quasi-Pfister $p$-form (over the corresponding field of the Knebusch splitting tower of $\phi)$. The sequence of integers $\left(\mathfrak{d}_{0}(\phi), \ldots, \mathfrak{d}_{h(\phi)}(\phi)\right)$ will be denoted by $\mathfrak{d}(\phi)$. As per Lemma 2.42 (and the proof of Proposition 2.39), we have:

Lemma 2.43. Let $\phi$ be a quasilinear $p$-form over $F$ and let $d=h(\phi)-h_{\mathrm{qp}}(\phi)$. Then $\mathfrak{d}(\phi)=\left(\mathfrak{d}_{0}(\phi), \ldots, \mathfrak{d}_{h_{\mathrm{qp}}(\phi)-1}(\phi), d, d-1, \ldots, 1,0\right)$.

We also, however, have the following information concerning the "nontrivial part" of the sequence $\mathfrak{d}(\phi)$ :

Lemma 2.44. Let $\phi$ be a quasilinear p-form over $F$. Then $\mathfrak{d}_{0}(\phi) \leq \cdots \leq \mathfrak{d}_{h_{\mathrm{qp}}(\phi)}$.

Proof. We may assume that $\phi$ is anisotropic and not split. We need to show that if $\phi$ is not similar to a quasi-Pfister $p$-form, then $\mathfrak{d}_{1}(\phi) \geq \mathfrak{d}_{0}(\phi)$. By Lemma 2.34 it will be sufficient to check that $\phi_{\text {sim }}$ remains anisotropic over $F(\phi)$. Suppose otherwise. Then, by Corollary 2.32(1), we have $N(\phi) \subseteq G(\phi)$. By Corollary 2.15 it follows that $N(\phi)=G(\phi)$, or, equivalently, that $\phi_{\text {nor }} \simeq \phi_{\text {sim }}$ (see Lemma 2.2). But, in view of Lemma 2.11 and Corollary 2.18, this implies that $\phi$ is similar to a quasi-Pfister $p$-form, thus contradicting our assumption. The lemma follows.

In particular, since $\mathfrak{i}_{r}(\phi)=\operatorname{dim} \phi_{r}-\operatorname{dim} \phi_{r-1}$ for all $1 \leq r \leq h(\phi)$ (see Remarks 2.36(2)), we obtain the following result concerning the integers $\mathfrak{i}_{r}(\phi)$ :

Corollary 2.45. Let $\phi$ be a quasilinear $p$-form over $F$. Then we have $\mathfrak{i}_{r}(\phi) \equiv 0$ $\left(\bmod \mathfrak{d}_{r-1}(\phi)\right)$ for all $1 \leq r \leq h_{\mathrm{qp}}(\phi)$.

2L. Some examples. We now conclude this section with two basic computations which will be needed in the sequel, beginning with:

Lemma 2.46. Let $\phi$ be an anisotropic quasilinear p-form over $F$ and let $\psi=$ $\phi_{F(T)} \perp\langle T\rangle$, where $T$ is an algebraically independent variable over $F$. Then $\mathfrak{i}(\psi)=\left(1, \mathfrak{i}_{1}(\phi), \mathfrak{i}_{2}(\phi), \ldots, \mathfrak{i}_{h(\phi)}(\phi)\right)$ and $\mathfrak{d}(\psi)=\left(0, \mathfrak{d}_{0}(\phi), \mathfrak{d}_{1}(\phi), \ldots, \mathfrak{d}_{h(\phi)}(\phi)\right)$.

Proof. The form $\psi$ is clearly anisotropic. Now, the field $F(T)(\psi)$ is $F$-isomorphic to a purely transcendental extension of $F$ (see the presentation of Remarks 2.6(1), for example). In particular, $\phi_{F(T)(\psi)}$ is anisotropic (Lemma 2.26), and so $\mathfrak{i}_{1}(\psi)=1$ and $\psi_{1} \simeq \phi_{F(T)(\psi)}$ (see Remark 2.5). Since $F(T)(\psi)$ is purely transcendental over $F$, the first statement now follows immediately from Lemma 2.26. In a similar way, Lemma 2.35 implies that $\mathfrak{d}_{r}(\psi)=\mathfrak{d}_{r-1}(\phi)$ for all $1 \leq r \leq h(\psi)$. Thus, to prove the second statement, it only remains to check that $\mathfrak{d}_{0}(\psi)=0$. But, since $\mathfrak{i}_{1}(\psi)=1$, this is an immediate consequence of Corollary 2.45.

Given this result, we can give an example of a quasilinear $p$-form whose (full) splitting pattern is not determined by its Knebusch splitting pattern: 
Example 2.47 (see [Hoffmann and Laghribi 2004, Example 8.15]). Let $T=$ $\left(T_{1}, \ldots, T_{n+1}\right)$ be a tuple of algebraically independent variables over a field $F_{0}$ of characteristic $p$, and let $F=F_{0}(T)$. Consider the form $\phi=\left\langle\left\langle T_{1}, \ldots, T_{n}\right\rangle\right\rangle \perp\left\langle T_{n+1}\right\rangle$ over $F$. By Lemma 2.46 and Proposition 2.39, we have

$$
\mathfrak{i}(\phi)=\left(1, p^{n}-p^{n-1}, p^{n-1}-p^{n-2}, \ldots, p^{2}-p, p-1\right),
$$

so that $\mathrm{j}_{r}(\phi)=p^{n}-p^{n-r+1}+1$ for all $1 \leq r \leq n+1$. On the other hand, the full splitting pattern of $\phi$ also contains all the integers $p^{n}-p^{n-r+1}(1 \leq r \leq n+1)$. Indeed, if $\left(L_{S}\right)$ denotes the Knebusch splitting tower of $\left\langle\left\langle a_{1}, \ldots, a_{n}\right\rangle_{L}\right.$, then we clearly have $\mathfrak{i}_{0}\left(\phi_{L_{r-1}}\right)=p^{n}-p^{n-r+1}$ for all $1 \leq r \leq n+1$ (again, we are using Lemma 2.26 and Proposition 2.39 here).

Our second computation is the following:

Lemma 2.48. Let $\phi$ be a quasilinear $p$-form over $F$. Let $\psi=\left\langle\left\langle T_{1}, \ldots, T_{n}\right\rangle\right\rangle \otimes \phi_{F(T)}$, where $T=\left(T_{1}, \ldots, T_{n}\right)$ is a tuple of algebraically independent variables over $F$. Then $\mathfrak{i}(\psi)=\left(p^{n} \mathfrak{i}_{1}(\phi), \ldots, p^{n} \mathfrak{i}_{h(\phi)}(\phi), p^{n}-p^{n-1}, p^{n-1}-p^{n-2}, \ldots, p^{2}-p, p-1\right)$ and $\mathfrak{d}(\psi)=\left(\mathfrak{d}_{0}(\phi)+n, \mathfrak{d}_{1}(\phi)+n, \ldots, \mathfrak{d}_{h(\phi)-1}(\phi)+n, n, n-1, \ldots, 1,0\right)$.

Proof. It is enough to treat the case where $n=1$. To simplify the notation, let us write $T$ for the variable $T_{1}$ and $L$ for the rational function field $F(T)$. Now, by construction, we have $\operatorname{ndeg}(\psi)=p(\operatorname{ndeg}(\phi))$ (see Remark 2.10). In view of Corollary 2.40, it follows that $h(\psi)=h(\phi)+1$. Let $\left(L_{r}\right)$ and $\left(F_{r}\right)$ denote the Knebusch splitting towers of $\psi$ and $\phi$ respectively. We claim that, for every $0 \leq r \leq h(\phi)$, $\left(L_{r}\right)_{T}$ is $F$-isomorphic to a purely transcendental extension of $F_{r}$. The case where $r=0$ is evident. In general, we have $\left(L_{r}\right)_{T}=\left(L_{T}\right)_{r}$, where $\left(\left(L_{T}\right)_{r}\right)$ denotes the Knebusch splitting tower of $\psi_{L_{T}}$. But, since $\langle\langle T\rangle\rangle_{L_{T}} \sim\langle 1\rangle$, and since $L_{T}$ is purely transcendental over $F$ (the $r=0$ case), we have $\left(\psi_{L_{T}}\right)_{\text {an }} \simeq \phi_{L_{T}}$. By Remark 2.5, it follows that $\left(L_{r}\right)_{T}$ is $L$-isomorphic to a purely transcendental extension of the free composite $F_{r} \cdot L_{T}$. Again, since $L_{T}$ is purely transcendental over $F$, the claim follows. Given this, Proposition 2.30 and Lemma 2.26 together imply that

$$
\mathfrak{j}_{r}(\psi)=\mathfrak{i}_{0}\left(\psi_{L_{r}}\right)=p \mathfrak{i}_{0}\left(\phi_{\left(L_{r}\right)_{T}}\right)=p \mathfrak{i}_{0}\left(\phi_{F_{r}}\right)=p \mathfrak{j}_{r}(\phi)
$$

for all $0 \leq r \leq h(\phi)$, which proves the first statement of the lemma. Similarly, our claim, Proposition 2.30 and Lemma 2.35 together imply that

$$
\mathfrak{d}_{r}(\psi)=\mathfrak{d}_{0}\left(\psi_{L_{r}}\right)=\mathfrak{d}_{0}\left(\phi_{\left(L_{r}\right)_{T}}\right)+1=\mathfrak{d}_{0}\left(\phi_{F_{r}}\right)+1=\mathfrak{d}_{r}(\phi)+1
$$

for all $0 \leq r \leq h(\phi)$, and so the second statement also holds.

\section{An incompressibility theorem and related results}

In this section, we collect some of the farther-reaching results on the isotropy behavior of quasilinear $p$-forms over function fields of quasilinear $p$-hypersurfaces 
which have been obtained in recent years. These results will have an essential role to play in the sequel. We do not provide full details here, but the interested reader is referred to the original articles [Hoffmann and Laghribi 2004; Totaro 2008; Scully 2016] for further information.

3A. The incompressibility theorem. Let $\phi$ be an anisotropic quasilinear $p$-form of dimension $\geq 2$ over $F$ with associated quasilinear $p$-hypersurface $X_{\phi}$. As in [Scully 2013, §5], we define the Izhboldin dimension of $X_{\phi}$, denoted $\operatorname{dim}_{\mathrm{Izh}}\left(X_{\phi}\right)$, to be the integer $\operatorname{dim} X_{\phi}-\mathfrak{i}_{1}(\phi)+1$. The following result was proved in [loc. cit.]:

Theorem 3.1 [Scully 2013, Theorem 5.12]. Let $X$ be an anisotropic quasilinear p-hypersurface over $F$. Let $Y$ be an algebraic variety over $F$ such that $Y\left(F_{\mathrm{sep}}\right)=\varnothing$. If $\operatorname{dim} Y<\operatorname{dim}_{\text {Izh }}(X)$, then there cannot exist a rational map $X \rightarrow Y$.

Remarks 3.2. (1) In the case where $p=2$, Theorem 3.1 is due to Totaro [2008, Theorem 5.1]. In fact, if $X_{\phi}=\{\phi=0\}$ is an anisotropic projective quadric over a field $k$ of any characteristic, and if $Y$ is any complete $k$-variety possessing no closed points of odd degree, then it is known that the existence of a rational map $X \rightarrow Y$ necessarily implies that $\operatorname{dim} Y \geq \operatorname{dim}_{\mathrm{Izh}}\left(X_{\phi}\right)$, where $\operatorname{dim}_{\mathrm{Izh}}\left(X_{\phi}\right)=\operatorname{dim} X_{\phi}-\mathfrak{i}_{1}(\phi)+1$. This result was first proved by Karpenko and Merkurjev [2003, Theorem 4.1] (see also [Elman et al. 2008, Theorem 76.5]) in the case where $X_{\phi}$ is smooth, and was later extended by Totaro [loc. cit.] to the singular case.

(2) The special case where $Y$ is a closed subvariety of $X$ shows that the canonical dimension of $X$ (see Section 1 ) is equal to $\operatorname{dim}_{\mathrm{Izh}}(X)$ (the inequality $\operatorname{cdim}(X) \leq$ $\operatorname{dim}_{\mathrm{Izh}}(X)$ is trivial; see [Scully 2013, Corollary 5.14]).

In the remainder of this section, we will recall some of the main applications of Theorem 3.1 (and its proof). Here, we mention the following:

Corollary 3.3 (see [Scully 2016, Corollary 5.4]). Let $\phi$ and $\psi$ be anisotropic quasilinear $p$-forms of dimension $\geq 2$ over $F$, and let $\sigma \subset \phi$ be a subform of dimension $\leq \operatorname{dim} \psi-\mathfrak{i}_{1}(\psi)$. Then $\sigma_{F(\psi)} \subset\left(\phi_{F(\psi)}\right)_{\text {an. In particular, } \sigma_{F(}(\psi)}$ is anisotropic.

Proof. We trivially have $D\left(\sigma_{F(\psi)}\right) \subseteq D\left(\phi_{F(\psi)}\right)$. In light of Proposition 2.3, it therefore suffices to check that $\sigma_{F(\psi)}$ is anisotropic, or, equivalently, that there does not exist a rational map $X_{\psi} \rightarrow X_{\sigma}$. But, since $X_{\sigma}\left(F_{\text {sep }}\right)=\varnothing$ (see Lemma 2.26), this is an immediate consequence of Theorem 3.1.

In particular, we have the following fundamental observation:

Corollary 3.4. Let $\phi$ be an anisotropic quasilinear $p$-form of dimension $\geq 2$ over $F$ and let $\psi \subset \phi$ be a subform of codimension $\mathfrak{i}_{1}(\phi)$. Then $\phi_{1} \simeq \psi_{F(\phi)}$.

Proof. By Corollary 3.3, we have $\psi_{F(\phi)} \subset \phi_{1}$. Since both forms have the same dimension by hypothesis, the result follows. 
3B. Neighbors and near neighbors, I. Let $\psi$ and $\phi$ be anisotropic quasilinear $p$-forms of dimension $\geq 2$ over $F$. We will say that $\psi$ is a neighbor (resp. near neighbor) of $\phi$ if $\psi$ is similar to a subform of codimension $<\mathfrak{i}_{1}(\phi)$ (resp. codimension $\mathfrak{i}_{1}(\phi)$ ) of $\phi$. Our motivation here is the following extension of Corollary 3.4:

Lemma 3.5. Let $\phi$ and $\psi$ be anisotropic quasilinear $p$-forms over $F$ such that $\phi$ is anisotropic of dimension $\geq 2$ and $\psi$ is similar to a subform of $\phi$. Then $\left(\psi_{F(\phi)}\right)_{\text {an }}$ is similar to $\phi_{1}$ if and only if $\psi$ is a neighbor or near neighbor of $\phi$.

Proof. Without loss of generality, we may assume that $\psi \subset \phi$. Again, we trivially have $D\left(\psi_{F(\phi)}\right) \subseteq D\left(\phi_{F(\phi)}\right)=D\left(\phi_{1}\right)$. By Proposition 2.3, it therefore suffices to check that $\operatorname{dim}\left(\psi_{F(\phi)}\right)_{\text {an }}=\operatorname{dim} \phi-\mathfrak{i}_{1}(\phi)$ if and only if $\psi$ has codimension $\leq \mathfrak{i}_{1}(\phi)$ in $\phi$. The left-to-right implication here is trivial. Conversely, if $\psi$ has codimension $\leq \mathfrak{i}_{1}(\phi)$ in $\phi$, then we have $\operatorname{dim}\left(\psi_{F(\phi)}\right)_{\text {an }} \geq \operatorname{dim} \phi-\mathfrak{i}_{1}(\phi)$ by Theorem 3.1. Since the reverse inequality holds here by obvious dimension reasons, the lemma is proved.

Note here that while neighbors of $\phi$ become anisotropic over $F(\phi)$, its near neighbors do not (Corollary 3.3). This enables us to compute:

Proposition 3.6 (see [Scully 2013, Proposition 6.1]). Let $\phi$ and $\psi$ be anisotropic quasilinear $p$-forms of dimension $\geq 2$ over $F$ such that $\psi$ is a codimension-d neighbor of $\phi$. Then $\mathfrak{i}_{1}(\psi)=\mathfrak{i}_{1}(\phi)-d$.

3C. The ruledness theorem. Another key application of Theorem 3.1 is the following extension of Proposition 3.6, which shows in a precise way that anisotropic quasilinear $p$-hypersurfaces having first higher isotropy index larger than 1 are ruled.

Theorem 3.7 (see [Scully 2013, Theorem 7.6]). Let $\phi$ and $\psi$ be anisotropic quasilinear $p$-forms of dimension $\geq 2$ over $F$ such that $\psi$ is a codimension-d neighbor of $\phi$. Then $X_{\phi}$ is birationally isomorphic to $X_{\psi} \times_{F} \mathbb{P}^{d}$.

Proof. By Proposition 3.6, we have $\mathfrak{i}_{1}(\psi)=\mathfrak{i}_{1}(\phi)-d$. It is therefore enough to treat the case where $d=\mathfrak{i}_{1}(\psi)-1$, and this is covered by [Scully 2013, Theorem 7.6].

Remark 3.8. Again, in the case where $p=2$, this result is due to Totaro [2008, Theorem 6.4]. Unlike Theorem 3.1, however, the analogous assertion remains open for generically smooth quadrics (in any characteristic; see [Totaro 2008; 2009]).

It is worth mentioning the following explicitly:

Corollary 3.9. Let $\phi$ and $\psi$ be anisotropic quasilinear p-forms of dimension $\geq 2$ over $F$ such that $\mathfrak{i}_{1}(\phi)>1$ and $\psi$ is similar to a codimension-1 subform of $\phi$. Then we have an F-isomorphism of fields $F(\phi) \simeq F[\psi]$.

Proof. By Theorem 3.7, $F(\phi)$ is $F$-isomorphic to a degree-one purely transcendental extension of $F(\psi)$. In view of Remarks 2.6(3), the result follows. 
3D. Neighbors and near neighbors, II. Given Theorem 3.7, we extend Proposition 3.6 as follows:

Proposition 3.10 (see [Scully 2016, Proposition 6.2]). Let $\phi$ and $\psi$ be anisotropic quasilinear $p$-forms of dimension $\geq 2$ over $F$ such that $\psi$ is a codimension-d neighbor of $\phi$. Then we have $\mathfrak{i}(\psi)=\left(\mathfrak{i}_{1}(\phi)-d, \mathfrak{i}_{2}(\phi), \ldots, \mathfrak{i}_{h(\phi)}(\phi)\right)$ and $\mathfrak{d}(\psi)=$ $\left(\mathfrak{d}_{0}(\psi), \mathfrak{d}_{1}(\phi), \ldots, \mathfrak{d}_{h(\phi)}(\phi)\right)$.

Proof. By Theorem 3.7, $F(\phi)$ is $F$-isomorphic to a purely transcendental extension of $F(\psi)$. Thus, if $\left(F_{r}\right)$ and $\left(F(\phi)_{r}\right)$ denote the Knebusch splitting towers of $\psi$ and $\psi_{F(\phi)}$, respectively, then $F(\phi)_{r}$ is $F_{r}$-isomorphic to a purely transcendental extension of $F_{r+1}$ for every $0 \leq r \leq h\left(\psi_{F(\phi)}\right)$. In particular, we have $\mathfrak{i}_{r}\left(\psi_{F(\phi)}\right)=\mathfrak{i}_{r+1}(\psi)$ and $\mathfrak{d}_{r}\left(\psi_{F(\phi)}\right)=\mathfrak{d}_{r+1}(\psi)$ for any such $r$ by Lemmas 2.26 and 2.35, respectively. On the other hand, Lemma 3.5 shows that $\left(\psi_{F(\phi)}\right)_{\text {an }}$ is similar to $\phi_{1}$. Since $\mathfrak{i}_{r}(\phi)=\mathfrak{i}_{r-1}\left(\phi_{1}\right)$ and $\mathfrak{d}_{r}(\phi)=\mathfrak{d}_{r-1}\left(\phi_{1}\right)$ for all $1 \leq r \leq h(\phi)$, the proposition follows immediately.

Let $\phi$ be a quasilinear $p$-form over $F$. We say that $\phi$ is a quasi-Pfister $p$-neighbor (of $\pi$ ) if there exists a quasi-Pfister $p$-form $\pi$ over $F$ such that $\phi$ is similar to a subform of $\pi$ and $\operatorname{dim} \phi>\frac{1}{p} \operatorname{dim} \pi$. If $\phi$ is anisotropic, then it follows from Proposition 2.39 that $\phi$ is a quasi-Pfister $p$-neighbor if and only if it is a neighbor of some quasi-Pfister $p$-form in the sense of Section 3B. Forms of this type are of special importance in the general theory of quasilinear $p$-forms. Putting Lemma 2.11, Proposition 2.39, Corollary 2.40, Lemma 3.5 and Proposition 3.10 together, we obtain the following classification of anisotropic quasi-Pfister $p$-neighbors:

Corollary 3.11 (see [Scully 2016, Theorem 6.4]). Let $\phi$ be an anisotropic quasilinear $p$-form of dimension $\geq 2$ over $F$ and let $n$ be the smallest nonnegative integer such that $p^{n+1} \geq \operatorname{dim} \phi$. Then the following are equivalent:

(1) $\phi$ is a quasi-Pfister p-neighbor.

(2) $\phi$ is a neighbor of $\phi_{\text {nor. }}$.

(3) $\phi_{F\left(\phi_{\text {nor }}\right)}$ is isotropic.

(4) $\operatorname{ndeg}(\phi)=p^{n+1}$.

(5) $h(\phi)=n+1$.

(6) $\mathfrak{i}_{1}(\phi)=\operatorname{dim} \phi-p^{n}$ and $\mathfrak{i}_{2}(\phi)=p^{n}-p^{n-1}$.

(7) $\mathfrak{i}(\phi)=\left(\operatorname{dim} \phi-p^{n}, p^{n}-p^{n-1}, p^{n-1}-p^{n-2}, \ldots, p^{2}-p, p-1\right)$.

(8) $\phi_{1}$ is similar to a quasi-Pfister p-form (i.e., $\left.h_{\mathrm{qp}}(\phi) \leq 1\right)$.

Remark 3.12. In the case where $p=2$, this result was proved earlier by Hoffmann and Laghribi [2004, Theorem 8.1] using different methods. 
For arbitrary subforms, the situation is naturally more complicated, but we can nevertheless appeal to the following general result which was proved in [Scully 2016] (and whose proof again makes essential use of Theorems 3.1 and 3.7):

Proposition 3.13 (see [Scully 2016, Proposition 8.6]). Let $\phi$ and $\psi$ be anisotropic quasilinear $p$-forms of dimension $\geq 2$ over $F$ such that $\phi_{F(\psi)}$ is isotropic. Then either

(1) $\left(\psi_{r}\right)_{F_{r}(\phi)}$ is anisotropic for all $0 \leq r<h(\psi)$ and $\mathfrak{i}\left(\psi_{F(\phi)}\right)=\mathfrak{i}(\psi)$, or

(2) $\mathfrak{i}\left(\psi_{F(\phi)}\right)=\left(\mathfrak{i}_{1}(\psi), \ldots, \mathfrak{i}_{s-1}(\psi), \mathfrak{i}_{s}(\psi)+\mathfrak{i}_{s+1}(\psi), \mathfrak{i}_{s+2}(\psi), \ldots, \mathfrak{i}_{h(\psi)}(\psi)\right)$, where $s<h(\psi)$ is the smallest nonnegative integer such that $\left(\psi_{s}\right)_{F_{s}(\phi)}$ is isotropic.

Note here that in the special situation where $\psi$ is a neighbor of $\phi$, we are necessarily in case (2) with $s$ being equal to 0 . Since $\left(\psi_{F(\phi)}\right)_{\mathrm{an}} \simeq \phi_{1}$ in this instance (Lemma 3.5), we recover the computation of Proposition 3.10. By contrast, if $\psi$ is a near neighbor of $\phi$, then we can be in either of cases (1) and (2) (see Remark 3.15 below). Nevertheless, we still have $\psi_{F(\phi)} \simeq \phi_{1}$ (Corollary 3.4), and so we get:

Corollary 3.14 (see [Scully 2016, Corollary 6.10]). Let $\phi$ be an anisotropic quasilinear $p$-form of dimension $\geq 2$ over $F$ and let $\psi$ be a near neighbor of $\phi$. Then either

(1) $\left(\psi_{r}\right)_{F_{r}(\phi)}$ is anisotropic for all $0 \leq r<h(\psi)$ and $\mathfrak{i}(\psi)=\mathfrak{i}\left(\phi_{1}\right)$, or

(2) $\mathfrak{i}(\psi)=\left(\mathfrak{i}_{2}(\phi), \mathfrak{i}_{3}(\phi), \ldots, \mathfrak{i}_{s}(\phi), \mathfrak{i}_{s}(\psi), \mathfrak{i}_{s+1}(\phi)-\mathfrak{i}_{s}(\psi), \mathfrak{i}_{s+2}(\phi), \ldots, \mathfrak{i}_{h(\phi)}(\phi)\right)$, where $s<h(\psi)$ is the smallest positive integer such that $\left(\psi_{s}\right)_{F_{s}(\phi)}$ is isotropic.

Remark 3.15. As per the comments above, neither of cases (1) and (2) can be ruled out here. Indeed, case (1) describes the situation where $h(\psi)=h(\phi)-1$, while case (2) describes the situation where $h(\psi)=h(\phi)$. As the reader will easily verify using Corollary 2.40 , both these situations can arise in practice.

3E. A comparison result. We now conclude this section by recalling the following comparison result for isotropy indices of quasilinear quadratic forms which was obtained in [Scully 2016] with the help of Theorem 3.1:

Proposition 3.16 (see [Scully 2016, Theorem 7.13, Remark 9.1]). Assume that $p=2$. Let $\phi$ be an anisotropic quasilinear quadratic form of dimension $\geq 2$ over $F$ and let $L$ be a field extension of $F$ such that $\phi_{L}$ is not split. Then

$$
\mathfrak{i}_{0}\left(\phi_{L(\phi)}\right)-\mathfrak{i}_{1}(\phi) \geq \min \left\{\mathfrak{i}_{0}\left(\phi_{L}\right),\left[\frac{1}{2}\left(\operatorname{dim} \phi-\mathfrak{i}_{1}(\phi)+1\right)\right]\right\} .
$$

Remark 3.17. A similar statement also holds for $p>2$ (see [Scully 2016, Theorem 7.13]), but this will not be needed below.

Finally, it will be convenient to record here the following application of this result: 
Theorem 3.18 [Scully 2016, Theorem 9.2]. Let $\phi$ be an anisotropic quasilinear quadratic form of dimension $\geq 2$ over $F$ and write $\operatorname{dim} \phi=2^{n}+m$ for uniquely determined integers $n \geq 0$ and $1 \leq m \leq 2^{n}$. Then, for any field extension $L$ of $F$, we either have $\mathfrak{i}_{0}\left(\phi_{L}\right) \geq m$ or $\mathfrak{i}_{0}\left(\phi_{L}\right) \leq m-\mathfrak{i}_{1}(\phi)$.

Remark 3.19. Taking $L=F(\phi)$ here, we see that if $\mathfrak{i}_{1}(\phi)<m$, then $\mathfrak{i}_{1}(\phi) \leq m / 2$. This result will now be subsumed in our Theorem 1.3.

\section{A motivational example}

As a warm-up for the proof of our main result, we will now prove Proposition 1.4. Throughout this section, we assume that $p=2$. By a quasi-Pfister form, we will mean a quasi-Pfister 2-form. Our assumption on the prime $p$ is imposed for simplicity, but also because we will make use of the following fact already mentioned in the introduction, the analogue of which is unknown when $p>3$ (see [Scully 2016, §4.1]):

Lemma 4.1 (see [Hoffmann 2004, Corollary 7.22]). Let $\phi$ be an anisotropic quasilinear quadratic form of dimension $\geq 2$ over $F$ and let $L$ be a field extension of $F$ such that $\phi_{L}$ is isotropic. Then $\mathfrak{i}_{0}\left(\phi_{L}\right) \geq \mathfrak{i}_{1}(\phi)$.

Now, let $\phi$ be an anisotropic quasilinear quadratic form of dimension $\geq 2$ over $F$ and let $\psi \subset \phi$ be a subform of codimension $\mathfrak{i}_{1}(\phi)$. By Corollary 3.4, $\psi_{F(\phi)}$ is isomorphic to the first higher anisotropic kernel of $\phi$. In the next section, we will prove Theorem 1.5, which asserts that the latter form is divisible by a quasi-Pfister form of dimension $\geq \mathfrak{i}_{1}(\phi)$. Here, we will consider some special situations in which this divisibility property is already visible over the base field $F$. To this end, we will be interested in the following technical condition on the pair $(\phi, \psi)$ :

( $\star$ There exist elements $a \in D(\phi) \backslash\{0\}$ and $b \in D(\psi) \backslash\{0\}$ such that $a \neq c(b d+e f)$ for any $c, d, e, f \in D(\psi)$.

Example 4.2. If, in the above situation, we have $\operatorname{ndeg}(\psi)<\operatorname{ndeg}(\phi)$ (equivalently, if $h(\psi)<h(\phi)$; see Corollary 2.40), then ( $\star$ ) holds for the pair $(\phi, \psi)$. Indeed, in this case, $D(\phi)$ is (evidently) not contained in $c N(\psi)$ for any $c \in F$. Since $b d+e f \in N(\psi)$ for every $b, d, e, f \in D(\psi)$, the validity of $(\star)$ is immediately verified.

In light of Example 4.2, Proposition 1.4 is subsumed in the following result:

Proposition 4.3. Let $\phi$ be an anisotropic quasilinear quadratic form of dimension $\geq 2$ over $F$ and let $\psi \subset \phi$ be subform of codimension $\mathfrak{i}_{1}(\phi)$ such that the pair $(\phi, \psi)$ satisfies $(\star)$. Then there exist a quasi-Pfister form $\pi$, a subform $\sigma \subset \pi$, an element $\lambda \in D(\phi)$ and a form $\tau$ over $F$ such that $\psi \simeq \pi \otimes \tau$ and $\phi \simeq \psi \perp \lambda \sigma$. 
Proof. Let $b \in D(\psi) \backslash\{0\}$ be as in $(\star)$. Then $(\star)$ also holds for the pair $(b \phi, b \psi)$. If this were not the case, then, for every $a \in D(\phi)$, we could find $c, d, e, f \in D(\psi)$ such that $b a=b c\left(b^{3} d+b e b f\right)$, or, equivalently, such that $a=b^{2} c(b d+e f)$. But, since $D(\psi)$ is an $F^{2}$-linear subspace of $F$, we have $b^{2} c \in D(\psi)$, and so this would contradict the fact that $(\star)$ holds for the original pair $(\phi, \psi)$. Since the exchange $(\phi, \psi) \rightarrow(b \phi, b \psi)$ does not affect the statement of the proposition, we can therefore assume that $b=1$. In other words, we can assume that $1 \in D(\psi)$ and that:

$\left(\star^{\prime}\right)$ There exists an element $a \in D(\phi) \backslash\{0\}$ such that $a \neq c(d+e f)$ for any $c, d, e, f \in D(\psi)$.

Let us fix $a \in D(\phi) \backslash\{0\}$ as in $\left(\star^{\prime}\right)$. We will now prove that the statement of the proposition holds with $\lambda=a$. First, we note that $\left(\star^{\prime}\right)$ implies:

(1) $a \notin D(\psi)$.

(2) $\psi_{F_{a u}}$ is anisotropic for every $u \in D(\psi) \backslash\{0\}$.

Indeed, if $a$ were in $D(\psi)$, then we could contradict ( $\left.\star^{\prime}\right)$ by taking $c=1, d=a$ and $e=f=0$. Similarly, if $\psi_{F_{a u}}$ were isotropic in (2), then (since $\psi$ is anisotropic) we could find nonzero elements $x, y \in D(\psi)$ such that $a u=x y$ (see Lemma 2.27(2)); taking $c=x^{-1}, d=0, e=y$ and $f=u$, this would again contradict $\left(\star^{\prime}\right)$.

Now, (1) implies that we have $\psi \perp\langle a\rangle \subset \phi$. If $\mathfrak{i}_{1}(\phi)=1$, then the latter inclusion is an isomorphism, and the statement of the proposition holds with $\pi=\sigma=\langle 1\rangle$ and $\tau=\psi$. Assume now that $\mathfrak{i}_{1}(\phi)>1$. Since $1, a \in D(\phi), \phi_{F_{a}}$ is isotropic by Lemma 2.27(2). By Lemma 4.1, it then follows that $\mathfrak{i}_{0}\left(\phi_{F_{a}}\right) \geq \mathfrak{i}_{1}(\phi)$. On the other hand, (2) (with $u=1 \in D(\psi)$ ) shows that $\psi_{F_{a}}$ is anisotropic, and, since $\operatorname{dim} \psi=\operatorname{dim} \phi-\mathfrak{i}_{1}(\phi)$, we conclude that $\left(\phi_{F_{a}}\right)_{\text {an }} \simeq \psi_{F_{a}}$. In other words, we have $D\left(\phi_{F_{a}}\right)=D\left(\psi_{F_{a}}\right)=D(\psi)+a D(\psi)$ (where the latter equality holds by Lemma 2.27(1)). By Lemma 2.2, it follows that we can write $\phi \simeq \psi \perp\langle a\rangle \perp a \sigma^{\prime}$ for some form $\sigma^{\prime} \subset \psi$. Let $\sigma=\langle 1\rangle \perp \sigma^{\prime}$, so that $\phi \simeq \psi \perp a \sigma$. As $1 \in D(\psi)$, we have $\sigma \subset \psi$. Since $\sigma$ is anisotropic and represents 1 , Lemma 2.11 shows that $\sigma \subset \sigma_{\text {nor }}$. Thus, in order to complete the proof, it will be enough to prove that $\psi$ is divisible by $\pi=\sigma_{\text {nor }}$. By Corollary 2.20, this amounts to showing that $D(\sigma) \subset G(\psi)$. Let $x \in D(\sigma) \backslash\{0\}$. In order to show that $x \in G(\psi)$, we must check that $x y \in D(\psi)$ for all $y \in D(\psi)$ (see Lemma 2.13). If $y=0$, then there is nothing to prove. Suppose now that $y \neq 0$. Then, by Lemma 2.27(2), $\phi_{F_{a y}}$ is isotropic. On the other hand, $\psi_{F_{a y}}$ is anisotropic by (2). Using Lemma 4.1 in the same way as before, we see that $\left(\phi_{F_{a y}}\right)_{\mathrm{an}} \simeq \psi_{F_{a y}}$, or, equivalently, that $D\left(\phi_{F_{a y}}\right)=D\left(\psi_{F_{a y}}\right)=D(\psi)+a y D(\psi)$. But, letting $u=\sqrt{a y}$, we have $x y=\left(a^{-1} u\right)^{2} a x \in D\left(\phi_{F_{a y}}\right)$. In particular, we can find $u, v \in D(\psi)$ such that $x y=u+a y v$. To complete the proof, it now only remains to show that $v=0$. But, if $v \neq 0$, then we have $a=v^{-1}\left(x+u y^{-1}\right)$, and we obtain a contradiction to $\left(\star^{\prime}\right)$ by taking $c=v^{-1}, d=x, e=u$ and $f=y^{-1}$. The result follows. 
In general, it is not always possible to find a subform $\psi \subset \phi$ of codimension $\mathfrak{i}_{1}(\phi)$ such that the pair $(\phi, \psi)$ satisfies condition $(\star)$. Indeed, note that if $\mathfrak{i}_{1}(\phi)=2$ in the situation of Proposition 4.3 , then $\phi$ is divisible by the binary (i.e., 2-dimensional) form $\sigma$. The following example, which is directly analogous to an example of Vishik from the characteristic $\neq 2$ theory of quadratic forms, shows that there exist anisotropic quasilinear quadratic forms which have first higher isotropy index equal to 2 , but which are not divisible by a binary form: let $a, b, c, d, e$ be algebraically independent variables over a field $F_{0}$ of characteristic 2, let $F=F_{0}(a, b, c, d, e)$ and consider the anisotropic $F$-form $\phi=\langle\langle a b, a c, a d\rangle\rangle \perp b c d\langle 1, a b, a c, a d\rangle \perp e\langle a, b, c, d\rangle$.

Lemma 4.4 (Vishik; see [Totaro 2009, Lemma 7.1]). In the above situation, we have $\mathfrak{i}_{1}(\phi)=2$, but $\phi$ is not divisible by a binary form.

Proof. We will freely use some basic facts from the theory of symmetric bilinear forms over fields of characteristic 2. If $B$ is such a form, then $\phi_{B}$ will denote the totally singular quadratic form $v \mapsto B(v, v)$. For all other notation and terminology, the reader is referred to [Elman et al. 2008, Chapter I].

Let $\mathfrak{b}$ be the bilinear form $\langle\langle a b, a c, a d\rangle\rangle_{b} \perp b c d\langle 1, a b, a c, a d\rangle_{b} \perp e\langle a, b, c, d\rangle_{b}$ over $F$, so that $\phi=\phi_{\mathfrak{b}}$. As the reader will immediately verify, we have

$$
\mathfrak{b} \sim \mathfrak{c}:=\langle\langle a, b, c, d\rangle\rangle_{b} \perp\left\langle\langle e\rangle_{b} \otimes\langle a, b, c, d\rangle_{b},\right.
$$

where the symbol $\sim$ denotes Witt equivalence. Let $\pi=\langle\langle a, b, c, d\rangle\rangle_{b}$, and let $\pi^{\prime}$ denote the pure subform of $\pi$. Since $\operatorname{ndeg}\left(\phi_{\pi}\right)=16<32=\operatorname{ndeg}(\phi)$, Corollary 2.32(2) implies that $\pi_{F(\phi)}$ is anisotropic. At the same time, it follows from Remarks 2.6(4) and the definition of $\phi$ that $e \in D\left(\pi_{F(\phi)}^{\prime}\right)$. Thus, by [Elman et al. 2008, Lemma 6.1], there exists a 3-fold bilinear Pfister form $\eta$ over $F(\phi)$ such that $\pi_{F(\phi)} \simeq\left\langle\langle e\rangle_{b} \otimes \eta\right.$. In particular, $\mathfrak{c}_{F(\phi)}$ is divisible by $\left\langle\langle e\rangle_{b}\right.$, and so $\mathfrak{i}_{W}\left(\mathfrak{c}_{F(\phi)}\right)$ is even (see [Elman et al. 2008, Proposition 6.22]; $\mathfrak{i}_{W}$ denotes here the Witt index). Since $\operatorname{dim} \mathfrak{c}-\operatorname{dim} \mathfrak{b}=8 \equiv$ $0(\bmod 4)$, it follows that $\mathfrak{i}_{W}\left(\mathfrak{b}_{F(\phi)}\right)$ is also even. In particular, this shows that $\mathfrak{i}_{1}(\phi) \geq 2$ (see [Laghribi 2007, Proposition 5.15]), and to prove that $\mathfrak{i}_{1}(\phi)=2$, it suffices to find a field extension $L$ of $F$ such that $\phi_{L}$ is isotropic but $\mathfrak{i}_{0}\left(\phi_{L}\right) \leq 2$ (see Lemma 4.1). We claim that $L=F_{c d e}$ is such an extension. First, note that since $c d e=(b c d)\left(b^{-1} e\right)$ is a product of two nonzero elements of $D(\phi), \phi_{L}$ is isotropic by Lemma 2.27(2). On the other hand, it is easy to see that the codimension-2 subform $\psi=\langle\langle a b, a c, a d\rangle\rangle \perp b c d\langle 1, a b, a c, a d\rangle \perp e\langle c, d\rangle \subset \phi$ remains anisotropic over $L$. Indeed, since

$$
\psi_{L} \simeq\langle\langle a b, a c, a d\rangle\rangle \perp b c d\langle 1, a b, a c, a d\rangle \perp\langle d, c\rangle \subset\langle\langle a, b, c, d\rangle\rangle_{L},
$$

it suffices to check that $\langle\langle a, b, c, d\rangle\rangle$ remains anisotropic over $L$. But, since $c d e \notin$ $F^{2}(a, b, c, d)=N(\langle\langle a, b, c, d\rangle\rangle)$, this follows from Lemma 2.27(4). Since the anisotropy of $\psi_{L}$ readily implies that $\mathfrak{i}_{0}\left(\phi_{L}\right) \leq 2$, we have proved our claim. 
It now remains to check that $\phi$ is not divisible by a binary form. For the sake of contradiction, suppose instead that $\phi$ is divisible by $\langle\langle u\rangle\rangle$ for some $u \in F \backslash F^{2}$. We claim that $u \in F^{2}(a b, a c, a d)$. Again, let us assume that this is not the case. Then the quasi-Pfister form $\tau=\langle\langle a b, a c, a d\rangle\rangle$ remains anisotropic over $F_{u}$ by Lemma 2.27(4). Let $\sigma=\tau \perp\langle b c d\rangle \subset \phi$ and $\eta=\tau \perp\langle a e\rangle \subset \phi$. Since $\mathfrak{i}_{0}\left(\phi_{F_{u}}\right)=\frac{1}{2} \operatorname{dim} \phi=8$ (Lemma 2.27(6)), and since $\operatorname{dim} \sigma=\operatorname{dim} \eta=9$, both $\sigma_{F_{u}}$ and $\eta_{F_{u}}$ are necessarily isotropic. Since $\tau_{F_{u}}$ is anisotropic, this means that $b c d$, ae $\in D\left(\tau_{F_{u}}\right)=F^{2}(a b, a c, a d, u)$. But this implies that $F^{2}(a, b, c, d, e) \subseteq$ $F^{2}(a b, a c, a d, u)$, thus contradicting the fact that the elements $a, b, c, d, e$ are algebraically independent over $F_{0}$. This proves our claim, and so we can write $u=v+w$ for some $v \in D(\langle 1, a b, a c, a d\rangle)$ and $w \in D(\langle b c, b d, c d, a b c d\rangle)$. Now, Lemma 2.27(6) implies that $u \in G(\phi)$. In particular, applying Lemma 2.13 to the elements $a e, a c d, a b d \in D(\phi)$, we see that

(1) aеu $\in D(\phi)$,

(2) $a c d u \in D(\phi)$, and

(3) $a b d u \in D(\phi)$.

We can now complete the proof: first, note that we have aev $\in D(\phi)$, since $a e\langle 1, a b, a c, a d\rangle \simeq e\langle a, b, c, d\rangle \subset \phi$. By (1), this implies that $a e w \in D(\phi)$. Note however that $a e\langle b c, b d, c d, a b c d\rangle \simeq\langle a b c e, a b d e, a c d e, b c d e\rangle$, and the latter form does not represent any nonzero element of $D(\phi)$. It follows that $w=0$, and so $u \in D(\langle 1, a b, a c, a d\rangle)$. Next, consider the form $\rho=a c d\langle 1, a b, a c, a d\rangle \simeq$ $\langle a c d, b c d, c, d\rangle$. By (2), we have $a c d u \in D(\rho) \cap D(\phi)$. Since the elements $a, b, c, d, e$ are algebraically independent over $F_{0}$, direct inspection shows that the former intersection is equal to $\operatorname{acd} D(\langle 1, a b\rangle)$, and so $u \in D(\langle 1, a b\rangle)$. Finally, we can use (3) in a similar way to show that $u \in F^{2}$, thus providing us with the needed contradiction. The lemma is proved.

In fact, Vishik's example shows more: it is generally not possible to find a subform $\psi \subset \phi$ of codimension $\mathfrak{i}_{1}(\phi)$ such that the pair $(\phi, \psi)$ satisfies condition $(\star)$, even after making arbitrary rational extensions of $F$ - this stronger assertion follows from Lemma 2.35. This leads us to consider the possibility that decompositions of the kind suggested by Proposition 4.3 may be found by passing to suitable transcendental extensions of the base field, and, ultimately, to our main result. Nevertheless, it is still interesting to ask for conditions on $\phi$ (or, more specifically, on the Knebusch splitting pattern of $\phi$ ) which automatically ensure the existence of the needed subform $\psi$. To this end, it is natural to look to the extremities where $\phi$ is "far from generic" (e.g., where $\mathfrak{i}_{1}(\phi)$ is "large"). The basic example is provided here by Proposition 2.39, which characterizes (scalar multiples of) anisotropic quasi-Pfister forms in terms of $\mathfrak{i}_{1}$, and one can hope that similar characterizations 
exist for "sufficiently simple" forms (see also Theorem 6.8 below). A better understanding of all these problems would have important implications for the study of symmetric bilinear forms of "low complexity" (e.g., of "small" height) in characteristic 2 .

\section{Main theorem}

We are now ready to give the proof of Theorem 1.5. In order to treat the case where $p>2$, the statement needs to be modified as follows:

Theorem 5.1. Let $\phi$ be an anisotropic quasilinear $p$-form of dimension $\geq 2$ over $F$ and let $s$ be the smallest nonnegative integer such that $p^{s} \geq \mathfrak{i}_{1}(\phi)$. If $\phi$ is not a quasi-Pfister p-neighbor, then $\phi_{1}$ is divisible by an s-fold quasi-Pfister p-form.

Remark 5.2. Nothing is lost here by assuming that $\phi$ is not a quasi-Pfister $p$-neighbor. Indeed, if $\phi$ is a quasi-Pfister $p$-neighbor, and $n$ denotes the smallest nonnegative integer such that $p^{n+1} \geq \operatorname{dim} \phi$, then $\phi_{1}$ is similar to an $n$-fold quasi-Pfister $p$-form by Corollary 3.11. If $p=2$, then we have $\mathfrak{i}_{1}(\phi) \leq \frac{1}{2} \operatorname{dim} \phi \leq 2^{n}$ by Proposition 2.39, so that $n \geq s$, where $s$ is the integer defined in the statement of the theorem. Note, however, that if $p>2$, then $n$ may be strictly smaller than $s$ (again, see Corollary 3.11). This explains why the additional hypothesis is needed here, but not in the statement of Theorem 1.5 (i.e., the case where $p=2$ ).

Proof. To simplify the notation, we will write $\mathfrak{i}_{1}$ instead of $\mathfrak{i}_{1}(\phi)$ in what follows. If $\mathfrak{i}_{1}=1$, then the statement of the theorem holds trivially. We therefore assume henceforth that $\mathfrak{i}_{1}>1$. After multiplying $\phi$ by a nonzero scalar if necessary, we may also assume that $1 \in D(\phi)$. In particular, we can find $a_{1}, \ldots, a_{n} \in F$ such that $\phi \simeq\left\langle 1, a_{1}, \ldots, a_{n}\right\rangle$. For the remainder of the proof, we let $\phi^{\prime}=\left\langle a_{1}, \ldots, a_{n}\right\rangle$, and we write $\phi^{\prime}(T)$ for the "generic value" of $\phi^{\prime}$, i.e., $\phi^{\prime}(T)=\sum_{i=1}^{n} a_{i} T_{i}^{p} \in F[T]$, where $T=\left(T_{1}, \ldots, T_{n}\right)$ is a tuple of algebraically independent variables over $F$. By Corollary 3.9, the function field $F(\phi)$ is $F$-isomorphic to $F\left[\phi^{\prime}\right]$, and may therefore be identified with $\operatorname{Frac}\left(F[T] /\left(\phi^{\prime}(T)\right)\right)$ (see Remarks 2.6(1)). Fixing this identification henceforth, we will write $\bar{f}$ for the image of a polynomial $f \in F[T]$ under the canonical $F$-algebra homomorphism $F[T] \rightarrow F(\phi)$. We will also write $m(f)$ for the multiplicity mult $\phi_{\phi^{\prime}(T)}(f)$ of $\phi^{\prime}(T)$ in $f$, i.e., the largest integer $k$ such that $f=\phi^{\prime}(T)^{k} h$ for some $h \in F[T]$. Note here that we have $\bar{f} \neq 0$ if and only if $m(f)=0$.

Now, let $\psi \subset \phi$ be any subform of codimension $\mathfrak{i}_{1}(\phi)$ such that $1 \in D(\psi)$. Then:

Lemma 5.3. In the above situation, we can find elements $g_{i, j} \in D\left(\psi_{F[T]}\right)\left(1 \leq i<i_{1}\right.$, $1 \leq j<p$ ) such that

$$
\phi_{F(T)} \simeq \psi_{F(T)} \oplus \phi^{\prime}(T)\left\langle 1, f_{1}, \ldots, f_{\mathfrak{i}_{1}-1}\right\rangle,
$$

where $f_{i}=\sum_{j=1}^{p-1} g_{i, j} \phi^{\prime}(T)^{j-1}$ for each $1 \leq i<\mathfrak{i}_{1}$. 
Proof. First, let us note that $\phi^{\prime}(T) \notin D\left(\psi_{F(T)}\right)$. Indeed, if $\psi_{F(T)}$ were to represent $\phi^{\prime}(T)$, then it would follow from Theorem 2.23 that $a_{1}, \ldots, a_{n} \in D(\psi)$. Since $1 \in D(\psi)$ by hypothesis, this would imply that $D(\phi) \subseteq D(\psi)$, or, equivalently, that $\phi \subset \psi$ (see Proposition 2.3), which is impossible for dimension reasons (recall here that $\mathfrak{i}_{1}>1$ by assumption). It follows that $\psi_{F(T)} \oplus\left\langle\phi^{\prime}(T)\right\rangle$ is anisotropic, and so $\psi_{F(T)} \oplus\left\langle\phi^{\prime}(T)\right\rangle \subset \phi_{F(T)}$ by Proposition 2.3. Now, the affine function field $F[\phi]$ may be identified (over $F$ ) with the field $K=F(T)_{\phi^{\prime}(T)}$ (see Remarks 2.6(1)). Since $F[\phi]$ is $F$-isomorphic to a purely transcendental extension of $F(\phi)$ (Remarks 2.6(2)), Corollary 3.4 and Lemma 2.26 together imply that $\psi_{K} \simeq\left(\phi_{K}\right)_{\text {an }}$. In particular, we have $D\left(\phi_{F(T)}\right) \subset D\left(\phi_{K}\right)=D\left(\psi_{K}\right)=$ $\sum_{j=0}^{p-1} D\left(\psi_{F(T)}\right) \phi^{\prime}(T)^{j}$ (where the last equality holds by Lemma 2.27(1)). Thus, by Lemma 2.2, we can complete the subform inclusion $\psi_{F(T)} \oplus\left\langle\phi^{\prime}(T)\right\rangle \subset \phi_{F(T)}$ to an isomorphism $\phi_{F(T)} \simeq \psi_{F(T)} \oplus\left\langle\phi^{\prime}(T)\right\rangle \oplus\left\langle f_{1}^{\prime}, \ldots, f_{\mathfrak{i}_{1}-1}^{\prime}\right\rangle$, where, for each $i$, we have $f_{i}^{\prime}=\sum_{j=0}^{p-1} g_{i, j} \phi^{\prime}(T)^{j}$ for some $g_{i, j} \in D\left(\psi_{F(T)}\right)$. Note, however, that every element of $D\left(\psi_{F(T)}\right)$ is (trivially) the ratio of an element of $D\left(\psi_{F[T]}\right)$ and a $p$-th power in $F[T]$. Since multiplying the $f_{i}^{\prime}$ by $p$-th powers in $F[T]$ does not change the $F(T)$-form $\left\langle f_{1}^{\prime}, \ldots, f_{\mathfrak{i}_{1}-1}^{\prime}\right\rangle$ up to isomorphism (see Lemma 2.2), we can arrange it so that the $g_{i, j}$ belong to $D\left(\psi_{F[T]}\right)$. Similarly, since subtracting elements of $D\left(\psi_{F(T)}\right)$ from the $f_{i}^{\prime}$ does not change the isomorphism class of $\psi_{F(T)} \oplus\left\langle f_{1}^{\prime}, \ldots, f_{\mathfrak{i}_{1}-1}^{\prime}\right\rangle$ (again, see Lemma 2.2), we can also arrange it so that $g_{i, 0}=0$ for all $i$. The remaining $g_{i, j}$ then satisfy the statement of the lemma.

Let us now fix elements $g_{i, j} \in D\left(\psi_{F[T]}\right)$ (and the associated polynomials $f_{i}$ ) satisfying the statement of Lemma 5.3. We are searching here for a sufficiently large quasi-Pfister divisor of $\phi_{1}$, and we would like to try to build this quasi-Pfister $p$-form from the elements $g_{i, 1}$. The basic point here is the following:

Lemma 5.4. In the above situation, we have $\overline{g_{i, 1}} b \in D\left(\phi_{1}\right)$ for all $b \in D(\psi)$ and all $1 \leq i<\mathfrak{i}_{1}$.

Proof. If $b=0$, then the statement is trivial. Let us now fix $b \in D(\psi) \backslash\{0\}$. We will need another lemma:

Lemma 5.5. In the above situation, there exist elements $s_{i, j} \in D\left(\psi_{F[T]}\right)$ and $t_{i, j} \in F[T] \backslash\{0\}\left(1 \leq i<\mathfrak{i}_{1}, 0 \leq j<p\right)$ such that, for every $1 \leq i<\mathfrak{i}_{1}$, we have:

(1) $b f_{i}=\sum_{j=0}^{p-1}\left(s_{i, j} / t_{i, j}^{p}\right)\left(\phi^{\prime}(T)^{j} / b^{j}\right)$ in $F(T)$.

(2) For each $0 \leq j<p$, at least one of $\overline{s_{i, j}}$ and $\overline{t_{i, j}}$ is nonzero.

Proof. Let $1 \leq i<\mathfrak{i}_{1}$, and consider the field $L=F(T)_{u}$, where $u=\phi^{\prime}(T) / b$. In view of Remarks 2.6(1), $L$ is $F$-isomorphic to the affine function field $F[\eta]$, where $\eta$ denotes the $F$-form $\langle b\rangle \perp \phi^{\prime}$. Now, since $b \in D(\psi)$, and since $\psi \subset \phi$, we have $D\left(\phi^{\prime}\right) \subseteq D(\eta) \subseteq D(\phi)$. In particular, if $\eta \not \nsucc \phi$, then it follows from Lemma 2.2 that 
$\eta_{\text {an }} \simeq \phi^{\prime}$. Either way, we see that $L$ is $F$-isomorphic to a degree-1 purely transcendental extension of $F(\phi)$ - in the first case, see Remarks 2.6(2); in the second, see Remarks 2.6(3) and Corollary 3.9. By Corollary 3.4 and Lemma 2.26, it follows that $\psi_{L} \simeq\left(\phi_{L}\right)_{\text {an }}$. In other words, we have $D\left(\phi_{L}\right)=D\left(\psi_{L}\right)=\sum_{j=0}^{p-1} D\left(\psi_{F(T)}\right) u^{j}$ (again, see Lemma 2.27(1) for the final equality). Now, since $u$ is a $p$-th power in $L$, we have $b f_{i}=\phi^{\prime}(T) f_{i} / u \in D\left(\phi_{L}\right)$. We can therefore write $b f_{i}=\sum_{i=0}^{p-1} q_{j} u^{j}$ for some $q_{j} \in D\left(\psi_{F(T)}\right)$. Since every element of $D\left(\psi_{F(T)}\right)$ is the quotient of an element of $D\left(\psi_{F[T]}\right)$ and a $p$-th power in $F[T]$, and since $u=\phi^{\prime}(T) / b$, this shows that we can find elements $s_{i, j} \in D\left(\psi_{F[T]}\right)$ and $t_{i, j} \in F[T] \backslash\{0\}$ such that (1) holds. Finally, since $\psi_{F(\phi)}$ is anisotropic, Proposition 2.33 implies that $m\left(s_{i, j}\right) \equiv 0(\bmod p)$ for all $0 \leq j<p$. For each such $j$, let $m_{j}=\min \left(m\left(s_{i, j}\right), p m\left(t_{i, j}\right)\right)$, and put $s_{i, j}^{\prime}=s_{i, j} / \phi^{\prime}(T)^{m_{j}}$ and $t_{i, j}^{\prime}=t_{i, j} / \phi^{\prime}(T)^{m_{j} / p}$. Then, by Theorem 2.23, we again have $s_{i, j}^{\prime} \in D\left(\psi_{F[T]}\right)$. Thus, replacing $s_{i, j}$ by $s_{i, j}^{\prime}$ and $t_{i, j}$ by $t_{i, j}^{\prime}$ (for each $j$ ), we arrive at the situation where, for any $j$, either $m\left(s_{i, j}\right)=0$ or $m\left(t_{i, j}\right)=0$. In other words, at least one of $\overline{i_{i, j}}$ and $\overline{t_{i, j}}$ is nonzero, as we wanted.

Returning now to the proof of Lemma 5.4, let $s_{i, j} \in D\left(\psi_{F[T]}\right)$ and $t_{i, j} \in F[T] \backslash\{0\}$ be as in Lemma 5.5. In particular, we have the equation

$$
\sum_{l=1}^{p-1} b g_{i, l} \phi^{\prime}(T)^{l-1}=b f_{i}=\sum_{j=0}^{p-1} \frac{s_{i, j}}{t_{i, j}^{p}} \frac{\phi^{\prime}(T)^{j}}{b^{j}}
$$

in $F(T)$. Clearing denominators, we obtain

$$
\prod_{k} t_{i, k}^{p} \sum_{l=1}^{p-1} b g_{i, l} \phi^{\prime}(T)^{l-1}=\sum_{j=0}^{p-1} \prod_{k \neq j} t_{i, k}^{p} s_{i, j} \frac{\phi^{\prime}(T)^{j}}{b^{j}} .
$$

Now, we claim that, for all $0 \leq j<p$, we have $\overline{t_{i, j}} \neq 0$, or, equivalently, $m\left(t_{i, j}\right)=0$. To see this, let $m=\min \left\{\sum_{k \neq j} m\left(t_{i, k}\right) \mid 0 \leq j<p\right\}$. Then our claim amounts to the assertion that $m=\sum_{k=0}^{p-1} m\left(t_{i, k}\right)$. Suppose that this is not the case, and let $0 \leq j<p$ be minimal so that $\sum_{k \neq j} m\left(t_{i, k}\right)=m$. Then, reducing both sides of (5-1) modulo $\phi^{\prime}(T)^{p m+j+1}$, we see that $s_{i, j} \equiv 0\left(\bmod \phi^{\prime}(T)\right)$. In other words, we have $\overline{s_{i, j}}=0$. By the choice of the $s_{i, j}$ and $t_{i, j}$, this implies that $\overline{t_{i, j}} \neq 0$, or, equivalently, that $m\left(t_{i, j}\right)=0$. But then $m=\sum_{k \neq j} m\left(t_{i, k}\right)=\sum_{k=0}^{p-1} m\left(t_{i, k}\right)$, which contradicts our assumption. The claim is therefore proved, and so, reducing (5-1) modulo $\phi^{\prime}(T)$ and dividing through by $\prod_{k} \overline{t_{i, k}} p$, we obtain the equality $\overline{g_{i, 1}} b=\overline{s_{i, 0}} / \overline{t_{i, 0}} p$ in $F(\phi)$. As $s_{i, 0} \in D\left(\psi_{F[T]}\right)$, this shows that $\overline{g_{i, 1}} b \in D\left(\psi_{F(\phi)}\right)$. But since $\psi_{F(\phi)} \simeq \phi_{1}$, we have $D\left(\psi_{F(\phi)}\right)=D\left(\phi_{1}\right)$, and the lemma is therefore proved.

Continuing with the proof of Theorem 5.1, let us now choose elements $g_{i, j}$ in $D\left(\psi_{F[T]}\right)$ as in the statement of Lemma 5.3 so that the integer $\sum_{i=1}^{\mathfrak{i}_{1}-1} \operatorname{deg}_{T_{1}}\left(g_{i, 1}\right)$ is minimal, where $\operatorname{deg}_{T_{1}}(g)$ denotes the degree of any $g \in F[T]$ viewed as an element 
of the ring $F\left(T_{2}, \ldots, T_{n}\right)\left[T_{1}\right]$, i.e., as a polynomial in the single variable $T_{1}$ (with the added convention that $\left.\operatorname{deg}_{T_{1}}(0)=0\right)$. Consider the form $\sigma=\left\langle 1, \overline{g_{1,1}}, \ldots, \overline{g_{i_{1}-1,1}}\right\rangle$ over $F(\phi)$. The final step in the proof of the theorem will be to prove the following statement:

Lemma 5.6. In the above situation, $\sigma$ is anisotropic.

Before proving the lemma, let us explain how this concludes the proof of Theorem 5.1. First, we claim that $\phi_{1}$ is divisible by the quasi-Pfister $p$-form $\sigma_{\text {nor }}$. By Corollary 2.20, this amounts to checking that $D(\sigma) \subseteq G\left(\phi_{1}\right)$. Since $G\left(\phi_{1}\right)$ is a subfield of $F$ containing $F^{p}$ (Corollary 2.15), it suffices to show here that $\overline{g_{i, 1}} \in G\left(\phi_{1}\right)$ for all $1 \leq i<\mathfrak{i}_{1}$. But by Lemma 2.13, this is equivalent to showing that, for all such $i$, we have $\overline{g_{i, 1}} D\left(\phi_{1}\right) \subseteq D\left(\phi_{1}\right)$. Since $D\left(\phi_{1}\right)=D\left(\psi_{F(\phi)}\right)$ is spanned as an $F(\phi)^{p}$-vector space by $D(\psi)$, this follows immediately from Lemma 5.4. The claim is therefore proved, and to finish the proof of the theorem, it only remains to check that $\operatorname{dim} \sigma_{\text {nor }} \geq p^{s}$. But, $\sigma$ is anisotropic by Lemma 5.6, and so $\sigma \subset \sigma_{\text {nor }}$ by Lemma 2.11. In particular, we have $\operatorname{dim} \sigma_{\text {nor }} \geq \operatorname{dim} \sigma=\mathfrak{i}_{1}$, which is precisely the assertion that $\operatorname{dim} \sigma_{\text {nor }} \geq p^{s}$ (because $\operatorname{dim} \sigma_{\text {nor }}$ is necessarily a power of $p$ ). Now, in order to prove Lemma 5.6, we need another auxiliary statement:

Lemma 5.7. If $\sigma$ is isotropic, then $p>2$, and there exist polynomials $g_{j} \in D\left(\psi_{F[T]}\right)$ $(1 \leq j<p)$ and an integer $2 \leq k<p$ such that:

(1) $\sum_{j=1}^{p-1} g_{j} \phi^{\prime}(T)^{j} \in D\left(\phi_{F[T]}\right)$.

(2) $m\left(g_{l}\right)>0$ for all $1 \leq l<k$.

(3) $m\left(g_{k}\right)=0$.

Proof. If $\sigma$ is isotropic, then since $\phi^{\prime}(T)$ is a Fermat-type polynomial of degree $p$, we can find an integer $1 \leq m \leq p$ and polynomials $h_{0}, \ldots, h_{\mathfrak{i}_{1}-1}, h \in F[T]$ such that:

(i) $h_{0}^{p}+g_{1,1} h_{1}^{p}+\cdots+g_{\mathfrak{i}_{1}-1,1} h_{\mathfrak{i}_{1}-1}^{p}=\phi^{\prime}(T)^{m} h$ in $F[T]$.

(ii) $\operatorname{deg}_{T_{1}}\left(h_{i}\right)<p$ for all $0 \leq i<\mathfrak{i}_{1}$.

(iii) $\overline{h_{i}} \neq 0$ for some $1 \leq i<\mathfrak{i}_{1}$.

First, let us note that we have $\phi^{\prime}(T)^{m} h \in D\left(\psi_{F[T]}\right)$ by (i) and the definition of the elements $g_{i, 1}$. Since $\psi_{F(\phi)}$ is anisotropic, it follows from Proposition 2.33 that $h=0$ or $m=p$. Either way, we can assume henceforth that $m=p$. Now, by (iii), there exists an $1 \leq l<\mathfrak{i}_{1}$ such that $h_{l} \neq 0$. Among all such integers $l$, let us fix one so that $\operatorname{deg}_{T_{1}}\left(g_{l, 1} h_{l}^{p}\right)$ is maximal. Consider now the polynomial $f=h_{0}^{p}+\sum_{k=1}^{\mathfrak{i}_{1}-1} f_{k} h_{k}^{p} \in$ $F[T]$, where the $f_{k}$ are as in the statement of Lemma 5.3. Since $h_{l} \neq 0$, Lemma 2.2 implies that $\left\langle 1, f_{1}, \ldots, f_{\mathfrak{i}_{1}-1}\right\rangle \simeq\left\langle 1, f_{1}, \ldots, f_{l-1}, f, f_{l+1}, \ldots, f_{\mathfrak{i}_{1}-1}\right\rangle$ as $F(T)$-forms. In particular, we have

$$
\phi_{F(T)} \simeq \psi_{F(T)} \oplus \phi^{\prime}(T)\left\langle 1, f_{1}, \ldots, f_{l-1}, f, f_{l+1}, \ldots, f_{\mathfrak{i}_{1}-1}\right\rangle .
$$


Now, by definition, $f=\sum_{j=1}^{p-1} g_{j}^{\prime} \phi^{\prime}(T)^{j-1}$, where $g_{1}^{\prime}=\phi^{\prime}(T)^{m} h \in D\left(\psi_{F[T]}\right)$ and $g_{j}^{\prime}=\sum_{k=1}^{\mathfrak{i}_{1}-1} g_{k, j} h_{k}^{p} \in D\left(\psi_{F[T]}\right)$ for all $2 \leq i<p$. Let $r=\min \left\{m\left(g_{j}^{\prime}\right) \mid 1 \leq j<p\right\}$. Since $g_{j}^{\prime} \in D\left(\psi_{F[T]}\right)$ for all $j$, and since $\psi_{F(\phi)}$ is anisotropic, another application of Proposition 2.33 shows that $r \equiv 0(\bmod p)$. In particular, for each $j \geq 1$, we have $g_{j}:=g_{j}^{\prime} / \phi^{\prime}(T)^{r} \in D\left(\psi_{F[T]}\right)$. In view of (5-2), it follows that the exchange $g_{l, j} \rightarrow g_{j}$ does not alter the statement of Lemma 5.3. By our choice of the $g_{i, j}$, we therefore have

$$
\operatorname{deg}_{T_{1}}\left(\phi^{\prime}(T)^{p-r} h\right)=\operatorname{deg}_{T_{1}}\left(g_{1}\right) \geq \operatorname{deg}_{T_{1}}\left(g_{l, 1}\right) .
$$

Now, we claim that the elements $g_{j}$ (together with an appropriate integer $k$ ) satisfy the conditions of the lemma. We have already seen here the validity of (1). At the same time, we have $m\left(g_{j}\right)=0$ for some $j \geq 1$ by construction. Thus, in order to prove the existence of an integer $k$ such that (2) and (3) are satisfied, we just need to check that $m\left(g_{1}\right)>0$. Recall again that we have $g_{1}=\phi^{\prime}(T)^{p-r} h$. If $h=0$, then there is nothing to prove. Suppose now that $h \neq 0$. By (i) and the choice of the integer $l$, we have $\operatorname{deg}_{T_{1}}\left(g_{l, 1} h_{l}^{p}\right) \geq \operatorname{deg}_{T_{1}}\left(\phi^{\prime}(T)^{p} h\right) \geq p^{2}+\operatorname{deg}_{T_{1}}(h)$. Since $\operatorname{deg}_{T_{1}}\left(h_{l}\right)<p$ (by (ii)), it follows that $\operatorname{deg}_{T_{1}}\left(g_{l, 1}\right)>\operatorname{deg}_{T_{1}}(h)$. In view of (5-3), we see that $r=0$ in this case. In particular, we have $g_{1}=\phi^{\prime}(T)^{p} h$, and so $m\left(g_{1}\right) \geq p>0$, as we wanted.

We are now ready to prove Lemma 5.6 and thus complete the proof of Theorem 5.1. If $p=2$, then the statement was already proved in Lemma 5.7. Suppose now that $p>2$, and assume for the sake of contradiction that $\sigma$ is isotropic. Let $g_{j}$ $(1 \leq j<p)$ and $k$ be as in the statement of Lemma 5.7. By condition (2) of the lemma, we can, for each $l<k$, write $g_{l}=\phi^{\prime}(T)^{m_{l}} h_{l}$ for some positive integer $m_{l}$ and some polynomial $h_{l}$. By a now familiar application of Proposition 2.33, we have $m_{l} \equiv 0(\bmod p)$ for every such $l$. In particular, the $m_{l}$ are all strictly larger than $k$. Now, by condition (1) of the lemma, the element

$\phi^{\prime}(T)^{k}\left(g_{k}+g_{k-1} \phi^{\prime}(T)+\cdots+g_{\mathfrak{i}_{1}-1} \phi^{\prime}(T)^{p-k-1}+h_{1} \phi^{\prime}(T)^{m_{1}-k}+\cdots+h_{k-1} \phi^{\prime}(T)^{m_{k-1}-k}\right)$

lies in $D\left(\phi_{F[T]}\right)$. Using the very same argument as that used to prove Lemma 5.4 above (and the fact that the integers $m_{l}-k(l<k)$ are all positive), one readily shows that $b^{k} \overline{g_{k}} \in D\left(\phi_{1}\right)$ for every $b \in D(\psi)$. Note, however, that $\overline{g_{k}} \neq 0$ by condition (3) of Lemma 5.7. Since $2 \leq k<p$, and since $\phi_{1} \simeq \psi_{F(\phi)}$, it follows from Lemma 2.22 that $\phi_{1}$ is a quasi-Pfister $p$-form. But, by Corollary 3.11, this in turn implies that $\phi$ is a quasi-Pfister $p$-neighbor, thus contradicting our original hypothesis. The lemma and theorem are therefore proved.

\section{First applications of the main theorem}

We now give the basic applications of Theorem 1.5. 
6A. Possible values of the Knebusch splitting pattern. Let $\phi$ be a quasilinear $p$-form of dimension $\geq 2$ over $F$. In the previous section we have shown that the first higher anisotropic kernel $\phi_{1}$ of $\phi$ is divisible by a quasi-Pfister $p$-form of dimension $\geq \mathfrak{i}_{1}(\phi)$, provided that $\phi_{\text {an }}$ is not a quasi-Pfister $p$-neighbor. In the terminology of Section $2 \mathrm{~K}$, this amounts to the assertion that if $h_{\mathrm{qp}}(\phi) \geq 2$, then $\mathfrak{d}_{1}(\phi) \geq \log _{p}\left(\mathfrak{i}_{1}(\phi)\right)$ (here we are also making use Corollary 3.11). By virtue of the inductive nature of the Knebusch splitting tower construction, we also obtain analogous restrictions on the higher isotropy indices $\mathfrak{i}_{r}(\phi)\left(2 \leq r<h_{\mathrm{qp}}(\phi)\right)$ in terms of the corresponding higher divisibility indices $\mathfrak{d}_{r}(\phi)$. Taking the observations of Section $2 \mathrm{~K}$ into account, our results may be summarized as follows:

Theorem 6.1. Let $\phi$ be a quasilinear $p$-form over $F$ and let $d=h(\phi)-h_{\mathrm{qp}}(\phi)$. Then:

(1) $\mathfrak{i}(\phi)=\left(\mathfrak{i}_{1}(\phi), \ldots, \mathfrak{i}_{h_{\mathrm{qp}}(\phi)}(\phi), p^{d}-p^{d-1}, p^{d-1}-p^{d-2}, \ldots, p^{2}-p, p-1\right)$.

(2) $\mathfrak{i}_{h_{\mathrm{qp}}}(\phi)=\operatorname{dim} \phi-\mathfrak{j}_{h_{\mathrm{qp}}(\phi)-1}(\phi)-p^{d}<p^{d+1}-p^{d}$.

(3) $\mathfrak{d}(\phi)=\left(\mathfrak{d}_{0}(\phi), \ldots, \mathfrak{d}_{h_{\mathrm{qp}}(\phi)-1}(\phi), d, d-1, \ldots, 1,0\right)$.

(4) $\mathfrak{d}_{0}(\phi) \leq \mathfrak{d}_{1}(\phi) \leq \cdots \leq \mathfrak{d}_{h_{\mathrm{qp}}(\phi)}=d$.

(5) $\mathfrak{i}_{r}(\phi) \equiv 0\left(\bmod p^{\mathfrak{d}_{r-1}(\phi)}\right)$ for all $1 \leq r<h_{\mathrm{qp}}(\phi)$.

(6) $\mathfrak{d}_{r}(\phi) \geq \log _{p}\left(\mathfrak{i}_{r}(\phi)\right)$ for all $1 \leq r<h_{\mathrm{qp}}(\phi)$.

(7) For every $1 \leq r<h_{\mathrm{qp}}(\phi), \mathfrak{i}_{r}(\phi)-1$ is the remainder of $\operatorname{dim} \phi-\mathfrak{j}_{r-1}(\phi)-1$ modulo $p^{\mathfrak{d}_{r}(\bar{\phi})}$.

Proof. Parts (1), (2), (3), (4) and (5) are the statements comprising Lemmas 2.42, 2.43, 2.44 and Corollary 2.45. Since $\mathfrak{i}_{r}(\phi)=\mathfrak{i}_{1}\left(\phi_{r-1}\right), \mathfrak{d}_{r}(\phi)=\mathfrak{d}_{1}\left(\phi_{r-1}\right)$ and $\operatorname{dim} \phi_{r-1}=\operatorname{dim} \phi-\mathfrak{j}_{r-1}(\phi)$ for all $1 \leq r \leq h(\phi)$, parts (6) and (7) follow immediately from Theorem 5.1 and Corollary 3.11.

In order to highlight the general shape of the Knebusch splitting pattern exposed by Theorem 6.1 , it is worth writing down the following result explicitly (here the notation $a \mid b$ means that $a$ divides $b$ ):

Corollary 6.2. Let $\phi$ be a quasilinear $p$-form over $F$. Then

$$
\mathfrak{i}_{1}(\phi) \leq p^{\mathfrak{d}_{1}(\phi)}\left|\mathfrak{i}_{2}(\phi) \leq p^{\mathfrak{d}_{2}(\phi)}\right| \cdots\left|\mathfrak{i}_{h_{\mathrm{qp}}(\phi)-1}(\phi) \leq p^{\mathfrak{d}_{h_{\mathrm{qp}}(\phi)-1}(\phi)}\right| \mathfrak{i}_{h_{\mathrm{qp}}(\phi)}(\phi) .
$$

Remark 6.3. In the special case where $p=2$, the chain of inequalities

$$
\mathfrak{i}_{1}(\phi) \leq \mathfrak{i}_{2}(\phi) \leq \cdots \leq \mathfrak{i}_{h_{\mathrm{qp}}(\phi)}(\phi)
$$

was previously obtained in [Scully 2016, Theorem 9.5] using Proposition 3.16. Here, we have given a more precise and natural explanation of this phenomenon.

We now show that, as far as the Knebusch splitting pattern is concerned, one cannot do any better than Theorem 6.1 in general: 
Proposition 6.4. Let $n$ be any positive integer. Suppose that we are given a nonnegative integer $k \leq n$ and two sequences $\left(\mathfrak{d}_{0}, \mathfrak{d}_{1}, \ldots, \mathfrak{d}_{k}=d\right)$ and $\left(\mathfrak{i}_{1}, \ldots, \mathfrak{i}_{k}\right)$ of $k+1$ and $k$ nonnegative integers, respectively, such that the following conditions hold:

(i) $\mathfrak{i}_{k}=n-\sum_{j=1}^{k-1} \mathfrak{i}_{j}-p^{d}<p^{d+1}-p^{d}$.

(ii) $\mathfrak{d}_{0} \leq \mathfrak{d}_{1} \leq \cdots \leq \mathfrak{d}_{k}=d$.

(iii) $1 \leq \mathfrak{i}_{r} \equiv 0\left(\bmod p^{\mathfrak{d}_{r-1}}\right)$ for all $1 \leq r<k$.

(iv) $\mathfrak{d}_{r} \geq \log _{p}\left(\mathfrak{i}_{r}\right)$ for all $1 \leq r<k$.

(v) For every $1 \leq r<k, \mathfrak{i}_{r}-1$ is the remainder of $n-\left(\sum_{j=1}^{r-1} \mathfrak{i}_{j}\right)-1$ modulo $p^{\mathfrak{d}_{r}}$. Then there exists a (purely transcendental) field extension $L$ of $F$ and an anisotropic quasilinear $p$-form $\phi$ of dimension $n$ over $L$ such that:

(1) $h_{\mathrm{qp}}(\phi)=k$.

(2) $h(\phi)=k+d$.

(3) $\mathfrak{d}(\phi)=\left(\mathfrak{d}_{0}(\phi), \ldots, \mathfrak{d}_{k-1}(\phi), d, d-1, \ldots, 1,0\right)$.

(4) $\mathfrak{d}_{r}(\phi) \geq \mathfrak{d}_{r}$ for all $0 \leq r<k$.

(5) $\mathfrak{i}(\phi)=\left(\mathfrak{i}_{1}, \ldots, \mathfrak{i}_{k}, p^{d}-p^{d-1}, p^{d-1}-p^{d-2}, \ldots, p^{2}-p, p-1\right)$.

Proof. We argue by induction on $k$. If $k=0$, then Proposition 2.39 shows that we can take $L=F(T)$ and $\phi=\left\langle\left\langle T_{1}, \ldots, T_{d}\right\rangle\right\rangle$, where $T=\left(T_{1}, \ldots, T_{d}\right)$ is a $d$-tuple of algebraically independent variables over $F$. Suppose now that $k>0$, and let $n^{\prime}=\left(n-\mathfrak{i}_{1}\right) / p^{\mathfrak{d}_{1}}$ and $\mathfrak{i}_{r}^{\prime}=\mathfrak{i}_{r+1} / p^{\mathfrak{d}_{1}}$ for all $1 \leq r<k$. By our hypotheses, these ratios are, in fact, positive integers. Setting $\mathfrak{d}_{r}^{\prime}=\mathfrak{d}_{r+1}-\mathfrak{d}_{1}$ for all $0 \leq r<k$, and putting $d^{\prime}=\mathfrak{d}_{k-1}^{\prime}$, conditions (i)-(v) then imply the following:

(i') $\mathfrak{i}_{k-1}^{\prime}=n^{\prime}-\sum_{j=1}^{k-2} \mathfrak{i}_{j}^{\prime}-p^{d^{\prime}}<p^{d^{\prime}+1}-p^{d^{\prime}}$.

(ii') $\mathfrak{d}_{0}^{\prime} \leq \mathfrak{d}_{1}^{\prime} \leq \cdots \leq \mathfrak{d}_{k-1}^{\prime}=d^{\prime}$.

(iii') $1 \leq \mathfrak{i}_{r}^{\prime} \equiv 0\left(\bmod p^{\mathfrak{d}_{r-1}^{\prime}}\right)$ for all $1 \leq r<k-1$.

(iv') $\mathfrak{d}_{r}^{\prime} \geq \log _{p}\left(\mathfrak{i}_{r}^{\prime}\right)$ for all $1 \leq r<k-1$.

$\left(\mathrm{v}^{\prime}\right)$ For every $1 \leq r<k-1, \mathfrak{i}_{r}^{\prime}-1$ is the remainder of $n^{\prime}-\left(\sum_{j=1}^{r-1} \mathfrak{i}_{j}^{\prime}\right)-1$ modulo $p^{\mathfrak{d}_{r}^{\prime}}$.

By the induction hypothesis, there exists a (purely transcendental) field extension $L_{0}$ of $F$ and an anisotropic quasilinear $p$-form $\psi$ of dimension $n^{\prime}$ over $L_{0}$ such that:

$\left(1^{\prime}\right) h_{\mathrm{qp}}(\psi)=k-1$.

(2') $h(\psi)=k-1+d^{\prime}$.

$\left(3^{\prime}\right) \mathfrak{d}(\psi)=\left(\mathfrak{d}_{0}(\psi), \mathfrak{d}_{1}(\psi), \ldots, \mathfrak{d}_{k-2}(\psi), d^{\prime}, d^{\prime}-1, \ldots, 1,0\right)$.

(4') $\mathfrak{d}_{r}(\psi) \geq \mathfrak{d}_{r}^{\prime}$ for all $0 \leq r<k-1$.

$\left(5^{\prime}\right) \mathfrak{i}(\psi)=\left(\mathfrak{i}_{1}^{\prime}, \ldots, \mathfrak{i}_{k-1}^{\prime}, p^{d^{\prime}}-p^{d^{\prime}-1}, p^{d^{\prime}-1}-p^{d^{\prime}-2}, \ldots, p^{2}-p, p-1\right)$. 
Consider now the form $\sigma=\psi_{L_{1}} \perp\left\langle T_{0}\right\rangle$ over the rational function field $L_{1}=L_{0}\left(T_{0}\right)$. By $\left(3^{\prime}\right),\left(5^{\prime}\right)$ and Lemma 2.46, we have:

(a) $\mathfrak{d}(\sigma)=\left(0, \mathfrak{d}_{0}(\psi), \mathfrak{d}_{1}(\psi), \ldots, \mathfrak{d}_{k-2}(\psi), d^{\prime}, d^{\prime}-1, \ldots, 1,0\right)$.

(b) $\mathfrak{i}(\sigma)=\left(1, \mathfrak{i}_{1}^{\prime}, \ldots, \mathfrak{i}_{k-1}^{\prime}, p^{d^{\prime}}-p^{d^{\prime}-1}, p^{d^{\prime}-1}-p^{d^{\prime}-2}, \ldots, p^{2}-p, p-1\right)$.

We would like to modify this further. Consider the product $\tau=\left\langle\left\langle T_{1}, \ldots, T_{\mathfrak{d}_{1}}\right\rangle\right\rangle \sigma_{L}$ over $L=L_{1}(T)$, where $T=\left(T_{1}, \ldots, T_{\mathfrak{d}_{1}}\right)$ is a $\mathfrak{d}_{1}$-tuple of algebraically independent variables over $L_{1}$. Then, by (a), (b) and Lemma 2.48, we have:

(c) $\mathfrak{d}(\tau)=\left(\mathfrak{d}_{1}, \mathfrak{d}_{1}(\psi)+\mathfrak{d}_{1}, \mathfrak{d}_{2}(\psi)+\mathfrak{d}_{1}, \ldots, \mathfrak{d}_{k-2}(\psi)+d_{1}, d, d-1, \ldots, 1,0\right)$.

(d) $\mathfrak{i}(\tau)=\left(p^{\mathfrak{d}_{1}}, \mathfrak{i}_{2}, \ldots, \mathfrak{i}_{k}, p^{d}-p^{d-1}, p^{d-1}-p^{d-2}, \ldots, p^{2}-p, p-1\right)$.

Now, by (iv), we have $\mathfrak{i}_{1}=p^{\mathfrak{d}_{1}}-s$ for some $0 \leq s<p^{\mathfrak{d}_{1}}$. By (ii) and (iii), $s$ is divisible by $p^{\mathfrak{d}_{0}}$. Let $\phi$ be any codimension-s subform of $\tau$ which is divisible by $\left\langle\left\langle T_{1}, \ldots, T_{\mathfrak{d}_{0}}\right\rangle\right\rangle$. Clearly $\phi$ is anisotropic, and by (c), (d) and Proposition 3.10, we have:

(e) $\mathfrak{d}(\phi)=\left(\mathfrak{d}_{0}(\phi), \mathfrak{d}_{1}(\psi)+\mathfrak{d}_{1}, \ldots, \mathfrak{d}_{k-2}(\psi)+\mathfrak{d}_{1}, d, d-1, \ldots, 1,0\right)$.

(f) $\mathfrak{i}(\phi)=\left(\mathfrak{i}_{1}, \mathfrak{i}_{2}, \ldots, \mathfrak{i}_{k}, p^{d}-p^{d-1}, p^{d-1}-p^{d-2}, \ldots, p^{2}-p, p-1\right)$.

The second statement shows that $\phi$ satisfies conditions (2) and (5). At the same time, since $\mathfrak{d}_{0}(\phi) \geq \mathfrak{d}_{0}$ by construction, and since $\mathfrak{d}_{2}(\psi)+\mathfrak{d}_{1} \geq \mathfrak{d}_{r}^{\prime}+\mathfrak{d}_{1}=\mathfrak{d}_{r+1}$ for all $0 \leq r<k-1$ by (4'), (e) shows that (3) and (4) are also satisfied. Finally, since $\mathfrak{i}_{k}<p^{d+1}-p^{d}$, Proposition 2.39 shows that $h_{\mathrm{qp}}(\phi)=k$, i.e., that (1) holds for $\phi$. The pair $(L, \phi)$ therefore has all the desired properties.

Remark 6.5. In general, it is not possible to arrange it so that $\mathfrak{d}_{r}(\phi)=\mathfrak{d}_{r}$ for all $0 \leq r<k$ in the statement of Proposition 6.4. For example, suppose that $p=2$, and take $n=2^{s+1}-2$ for some $s \geq 3, k=1, \mathfrak{d}_{0}=0, \mathfrak{d}_{1}=s, \mathfrak{i}_{0}=0, \mathfrak{i}_{1}=2^{s}-2$. As the reader will readily verify, these integers satisfy conditions (i)-(v) of the proposition. On the other hand, let $(L, \phi)$ be any pair consisting of a field extension $L$ of $F$ and an anisotropic form $\phi$ of dimension $2^{s+1}-2$ over $L$ such that $\mathfrak{i}_{1}(\phi)=2^{s}-2$. By Theorem 6.8 below (see also [Scully 2016, Theorem 9.6]), $\phi$ is necessarily a quasi-Pfister 2-neighbor, and therefore satisfies conditions (1)-(5) of the proposition (see Corollary 3.11). We claim, however, that $\mathfrak{d}_{0}(\phi)>0$, i.e., that $\phi$ is divisible by a binary form. To see this, note that there exists an anisotropic $(s+1)$-fold bilinear Pfister form $\pi$ over $L$ and a subform $\mathfrak{b} \subset \pi$ such that $\phi$ is given by the assignment $v \mapsto \mathfrak{b}(v, v)$. Since $\mathfrak{b}$ (being a codimension-2 subform of $\pi$ ) becomes split over the extension $K=L_{\operatorname{det}(\mathfrak{b})}$ (where det denotes the determinant), we have $\mathfrak{i}_{0}\left(\phi_{K}\right) \geq \frac{1}{2} \operatorname{dim} \phi$ (see [Laghribi 2007, Proposition 5.15]), whence $\phi$ is divisible by $\langle\langle\operatorname{det}(\mathfrak{b})\rangle\rangle(\operatorname{Lemma} 2.27(6))$.

Theorem 6.1 and Proposition 6.4 thus give a complete solution to the problem of determining the possible values of the Knebusch splitting pattern for quasilinear 
$p$-forms. In particular, we have an answer to Question 1.1 in the totally singular case. As noted in Example 2.47, the Knebusch and full splitting patterns need not agree in general for quasilinear $p$-forms. In Section 7 below, we will consider the problem of determining the possible values of the full splitting pattern in the case where $p=2$.

6B. Canonical dimensions of quasilinear p-hypersurfaces. Since the canonical dimension of an anisotropic quasilinear $p$-hypersurface is determined by the Knebusch splitting pattern of its underlying form (this is the basic fact underlying our proof of Theorem 5.1; see Theorem 3.1 and Remarks 3.2(2)), we also obtain a list of all restrictions on the possible values of the former invariant:

Theorem 6.6. Let $X$ be an anisotropic quasilinear p-hypersurface over $F$. Then there exists a nonnegative integer s such that:

(1) $p^{s}-1 \leq \operatorname{dim} X<p^{s}+\operatorname{cdim}(X)$.

(2) $\operatorname{cdim}(X) \equiv-1\left(\bmod p^{s}\right)$.

There are no further restrictions on $\operatorname{cdim}(X)$.

Proof. As per the above discussion, this follows readily from Theorems 3.1 and 6.1 and Proposition 6.4 (see also Remarks 3.2(2)).

Remarks 6.7. (1) We emphasize again that $\operatorname{cdim}(X)$ is to be understood here as the minimum dimension of the image of a rational self-map $X \rightarrow X$. For $p>3$, we do not know if this agrees with the definition given in [Elman et al. 2008, §90] (although this is certainly expected; see [Scully 2016, Question 4.4, Proposition 4.7]).

(2) The problem of determining all possible values of the canonical dimension for smooth (projective) hypersurfaces $X$ of prime degree $p>2$ in characteristic $\neq p$ remains open in general. Using a degree formula, Merkurjev [2003, §7.3] showed that if $\operatorname{dim} X \geq p^{n}-1$ for some nonnegative integer $n$, then we also have $\operatorname{cdim}(X) \geq p^{n}-1$ provided that $X$ has no points of degree prime to $p$. Little else is known. By way of specialization, our Theorem 6.6 may be of some use when considering specific examples over certain fields of characteristic zero.

6C. Quasilinear p-forms with maximal splitting. Let $\phi$ be an anisotropic quasilinear $p$-form of dimension $\geq 2$ over $F$ and write $\operatorname{dim} \phi=p^{n}+m$ for uniquely determined integers $n \geq 0$ and $1 \leq m \leq p^{n+1}-p^{n}$. By Theorem 6.1 (see also [Scully 2013, Corollary 6.8]), we have $\mathfrak{i}_{1}(\phi) \leq m$. If equality holds here, then we say that $\phi$ has maximal splitting. The basic examples of forms having this property are given by anisotropic quasi-Pfister $p$-neighbors (see Corollary 3.11). It is interesting to ask here to what extent this property characterizes quasi-Pfister $p$-neighbors. Given Theorem 5.1, we can now prove the following general result: 
Theorem 6.8. Let $\phi$ be an anisotropic quasilinear $p$-form of dimension $\geq 2$ over $F$ and let $n$ be the smallest nonnegative integer such that $p^{n+1} \geq \operatorname{dim} \phi$. If $\phi$ has maximal splitting, and if either

(1) $p>2$ and $\operatorname{dim} \phi>p^{n}+p^{n-1}$, or

(2) $p=2$ and $\operatorname{dim} \phi>2^{n}+2^{n-2}$,

then $\phi$ is a quasi-Pfister p-neighbor.

Proof. By Theorem 5.1, we may assume that $\mathfrak{d}_{1}(\phi) \geq \mathfrak{i}_{1}(\phi)$. Suppose first that $p>2$. Since $\phi$ has maximal splitting, we have $\mathfrak{i}_{1}(\phi)>p^{n-1}$ by (1), and so $\mathfrak{d}_{1}(\phi) \geq p^{n}$. On the other hand, we have $\operatorname{dim} \phi_{1}=\operatorname{dim} \phi-\mathfrak{i}_{1}(\phi)=p^{n}$ (see Remarks 2.36(2)). It follows that $\phi_{1}$ is similar to an $n$-fold quasi-Pfister $p$-form, and so $\phi$ is a quasi-Pfister $p$-neighbor by Corollary 3.11. If $p=2$, the same argument (and (2)) shows that $\phi_{1}$ is a form of dimension $2^{n}$ which is divisible by an $(n-1)$-fold quasi-Pfister 2-form. Since every binary form is similar to a quasi-Pfister 2 -form in this case, $\phi_{1}$ is, in fact, similar to an $n$-fold quasi-Pfister 2-form, and we now conclude as before.

Remark 6.9. The statement of Theorem 6.8 was originally conjectured in [Hoffmann 2004, Remark 7.32] (see also [Scully 2016, Question 7.6]). The result is the best possible, in the sense that one has examples of anisotropic quasilinear $p$-forms with maximal splitting which are not quasi-Pfister $p$-neighbors in every dimension omitted in the statement of the theorem (see [Hoffmann 2004, Example 7.31]). In the special case where $p=2$, Theorem 6.8 was previously established in [Scully 2016, Theorem 9.6] using Proposition 3.16. Here, we obtain a more natural explanation of this phenomenon by way of Theorem 5.1. Note that the $p=2$ case of the theorem is a direct analogue of a conjecture of Hoffmann in the theory of nonsingular quadratic forms which remains open, even over fields of characteristic different from 2 (see [Hoffmann 1995, §4; Izhboldin and Vishik 2000, Conjecture 1.6].

\section{Further remarks on the splitting of quasilinear quadratic forms}

Having determined all possible standard splitting patterns of quasilinear $p$-forms (Theorem 6.1, Proposition 6.4), we now turn our attention towards the problem of obtaining a similar result for the full splitting pattern. Here, we restrict our considerations to the case of quasilinear quadratic forms, where we can take direct inspiration from the following theorem of Vishik (which may be deduced from the existence of "excellent connections" in the integral Chow motives of anisotropic quadrics over fields of characteristic $\neq 2$; see [Vishik 2011, Theorem 1.3]):

Theorem 7.1 [Vishik 2011]. Let $\phi$ be an anisotropic quadratic form of dimension $\geq 2$ over a field $k$ of characteristic $\neq 2$. Let $\operatorname{dim} \phi-\mathfrak{i}_{1}(\phi)=2^{r_{1}}-2^{r_{2}}+\cdots+(-1)^{s-1} 2^{r_{s}}$ for uniquely determined integers $r_{1}>r_{2}>\cdots>r_{s-1}>r_{s}+1 \geq 1$. Let $1 \leq l \leq s$, and put $D_{l}=\sum_{i=1}^{l-1}(-1)^{i-1} 2^{r_{i}-1}+\epsilon(l) \sum_{j=l}^{s}(-1)^{j-1} 2^{r_{j}}$, where $\epsilon(l)=1($ resp. $\epsilon(l)=0)$ 
if $l$ is even (resp. odd). Then, for any field extension $L$ of $k$, we either have $\mathfrak{i}_{0}\left(\phi_{L}\right) \geq D_{l}+\mathfrak{i}_{1}(\phi)$ or $\mathfrak{i}_{0}\left(\phi_{L}\right) \leq D_{l}$.

Proof. This is nothing else but a restatement of [Vishik 2011, Proposition 2.6] in terms of Witt indices. To see how it may be derived from [Vishik 2011, Theorem 2.1] in further detail, we refer the reader to [Scully 2016, Proof of Theorem 1.2].

Examples 7.2. Let $\phi$ be an anisotropic quadratic form of dimension $\geq 2$ over a field $k$ of characteristic $\neq 2$, and write $\operatorname{dim} \phi=2^{n}+m$ for uniquely determined integers $n \geq 0$ and $1 \leq m \leq 2^{n}$.

(1) For $l=1$, Theorem 7.1 asserts that $\mathfrak{i}_{0}\left(\phi_{L}\right) \geq \mathfrak{i}_{1}(\phi)$ whenever $\phi_{L}$ is isotropic.

(2) For $l=2$, Theorem 7.1 asserts that, for any field extension $L$ of $k$, we either have $\mathfrak{i}_{0}\left(\phi_{L}\right) \geq m$ or $\mathfrak{i}_{0}\left(\phi_{L}\right) \leq m-\mathfrak{i}_{1}\left(\phi_{L}\right)$.

(3) For $l=s$, Theorem 7.1 asserts that, for any field extension $L$ of $k$, we either have $\mathfrak{i}_{0}\left(\phi_{L}\right) \geq \frac{1}{2}\left(\operatorname{dim} \phi+\mathfrak{i}_{1}(\phi)-2^{r_{s}}\right)$ or $\mathfrak{i}_{0}\left(\phi_{L}\right) \leq \frac{1}{2}\left(\operatorname{dim} \phi-\mathfrak{i}_{1}(\phi)-2^{r_{s}}\right)$. Taking $L=\bar{k}$ (so that $\mathfrak{i}_{0}\left(\phi_{L}\right)=\left[\frac{1}{2} \operatorname{dim} \phi\right]$ ), we see that $\mathfrak{i}_{1}(\phi) \leq 2^{r_{s}}$, which gives a proof of Hoffmann's Conjecture 1.2 in this setting (see [Vishik 2011, Theorem 2.5.])

We expect, in fact, that Theorem 7.1 extends verbatim to our setting. Henceforth, let us assume that $p=2$. We state the following:

Conjecture 7.3. Let $\phi$ be an anisotropic quasilinear quadratic form of dimension $\geq 2$ over $F$, and write $\operatorname{dim} \phi-\mathfrak{i}_{1}(\phi)=2^{r_{1}}-2^{r_{2}}+\cdots+(-1)^{s-1} 2^{r_{s}}$ for uniquely determined integers $r_{1}>r_{2}>\cdots>r_{s-1}>r_{s}+1 \geq 1$. Let $1 \leq l \leq s$, and put $D_{l}=\sum_{i=1}^{l-1}(-1)^{i-1} 2^{r_{i}-1}+\epsilon(l) \sum_{j=l}^{s}(-1)^{j-1} 2^{r_{j}}$, where $\epsilon(l)=1$ (resp. $\left.\epsilon(l)=0\right)$ if $l$ is even (resp. odd). If $L$ is any field extension of $F$, then we either have $\mathfrak{i}_{0}\left(\phi_{L}\right) \geq D_{l}+\mathfrak{i}_{1}(\phi)$ or $\mathfrak{i}_{0}\left(\phi_{L}\right) \leq D_{l}$.

This expectation is partly justified by:

Proposition 7.4. Conjecture 7.3 holds for $l \leq 2$.

Proof. As in Examples 7.2(1) (resp. (2)), the $l=1$ (resp. $l=2$ ) case is nothing else but Lemma 4.1 (resp. Theorem 3.18).

At present, we do not have a general approach to the $l>2$ case of Conjecture 7.3. Using Theorem 1.3, however, we can provide further evidence for the $l=s$ case. First, it is worth stating here the following lemma:

Lemma 7.5. In order to prove Conjecture 7.3, we may assume that $\mathfrak{i}_{1}\left(\phi_{L}\right) \geq \mathfrak{i}_{1}(\phi)$.

Proof. Suppose that the statement of the conjecture fails to hold. In other words, suppose that $\mathfrak{i}_{1}(\phi)>1$ and $\mathfrak{i}_{0}\left(\phi_{L}\right)=D_{l}+t$ for some $1 \leq t<\mathfrak{i}_{1}(\phi)$. Note that we necessarily have $r_{s}>0$ by Theorem 1.3. Now, let $\sigma=\left(\phi_{L}\right)_{\text {an }}$. Since $D(\sigma)$ is spanned by elements of $D(\phi)$, there exists a subform $\rho \subset \phi$ such that $\sigma \simeq \rho_{L}$ 
(see Lemma 2.2). Let $\psi$ be any codimension- $(t-1)$ subform of $\phi$ containing $\rho$. By construction, we have $D\left(\psi_{L}\right) \subseteq D\left(\phi_{L}\right)=D(\sigma)=D\left(\rho_{L}\right) \subseteq D\left(\psi_{L}\right)$, whence $D\left(\psi_{L}\right)=D(\sigma)$, or, equivalently, $\left(\psi_{L}\right)_{\text {an }} \simeq \sigma$. In particular, we have $\mathfrak{i}_{0}\left(\psi_{L}\right)=D_{l}+1$. On the other hand, since $t<\mathfrak{i}_{1}(\phi), \psi$ is a neighbor of $\phi$, and so $\operatorname{dim} \psi-\mathfrak{i}_{1}(\psi)=$ $\operatorname{dim} \phi-\mathfrak{i}_{1}(\phi)=2^{r_{1}}-2^{r_{2}}+\cdots+(-1)^{s-1} 2^{r_{s}}$ (Proposition 3.10). We therefore conclude that the statement of the conjecture also fails for the triple $(\psi, l, L)$. Since we are looking to produce a contradiction, we can replace $\phi$ by $\psi$ in order to arrive at the case where $t=1$. In this case, we claim that $\mathfrak{i}_{1}\left(\phi_{L}\right) \geq \mathfrak{i}_{1}(\phi)$. To see this, note first that $L(\phi)$ is $L$-isomorphic to a purely transcendental extension of $L(\sigma)$ (Remarks 2.6(3)). In view of Lemma 2.26, it follows that $\mathfrak{i}_{1}\left(\phi_{L}\right)=\mathfrak{i}_{1}\left(\sigma_{L(\phi)}\right)$. In particular, we have $\mathfrak{i}_{0}\left(\phi_{L(\phi)}\right)=\mathfrak{i}_{0}\left(\phi_{L}\right)+\mathfrak{i}_{0}\left(\sigma_{L(\phi)}\right)=\mathfrak{i}_{0}\left(\phi_{L}\right)+\mathfrak{i}_{1}\left(\phi_{L}\right)$. The statement of Proposition 3.16 may therefore be rewritten here as

$$
\mathfrak{i}_{1}\left(\phi_{L}\right)+\mathfrak{i}_{0}\left(\phi_{L}\right)-\mathfrak{i}_{1}(\phi) \geq \min \left\{\mathfrak{i}_{0}\left(\phi_{L}\right),\left[\frac{1}{2}\left(\operatorname{dim} \phi-\mathfrak{i}_{1}(\phi)+1\right)\right]\right\} .
$$

But, since $r_{s}>0$, we have $\mathfrak{i}_{0}\left(\phi_{L}\right)=D_{l}+1 \leq 2^{r_{1}-1}-2^{r_{2}-1}+\cdots+(-1)^{s-1} 2^{r_{s}-1}=$ $\frac{1}{2}\left(\operatorname{dim} \phi-\mathfrak{i}_{1}(\phi)\right)$. Inequality (7-1) therefore yields the desired assertion, and so the lemma is proved.

We can now prove:

Proposition 7.6. Conjecture 7.3 holds when $l=s$ and $L=F(Q)$ is the function field of any integral (affine or projective) quadric $Q$ over $F$.

Proof. In view of Lemma 2.26, the statement of the conjecture is stable under replacing $F$ by any separable extension of itself. We may therefore assume that $L$ is a purely inseparable quadratic extension of $F$. By Lemma 7.5, we may also assume that $\mathfrak{i}_{1}\left(\phi_{L}\right) \geq \mathfrak{i}_{1}(\phi)$. Suppose now that the statement fails to hold, so that $\mathfrak{i}_{1}(\phi)>1$ and $\mathfrak{i}_{0}\left(\phi_{L}\right)=D_{s}+t$ for some $1 \leq t<\mathfrak{i}_{1}(\phi)$. We then have

$$
\operatorname{dim}\left(\phi_{L}\right)_{\mathrm{an}}= \begin{cases}2^{r_{1}-1}-2^{r_{2}-1}+\cdots-2^{r_{s-2}-1}+2^{r_{s-1}-1}+\mathfrak{i}_{1}(\phi)-t & \text { if } s \text { is even, } \\ 2^{r_{1}-1}-2^{r_{2}-1}+\cdots-2^{r_{s-1}-1}+2^{r_{s}}+\mathfrak{i}_{1}(\phi)-t & \text { if } s \text { is odd. }\end{cases}
$$

Since $\mathfrak{i}_{1}\left(\phi_{L}\right) \geq \mathfrak{i}_{1}(\phi)$, and since $1 \leq \mathfrak{i}_{1}(\phi)-t<\mathfrak{i}_{1}(\phi)$, Theorem 1.3 implies that

$$
\mathfrak{i}_{1}\left(\phi_{L}\right) \geq \begin{cases}2^{r_{s-1}-1}+\mathfrak{i}_{1}(\phi)-t & \text { if } s \text { is even, } \\ 2^{r_{s}}+\mathfrak{i}_{1}(\phi)-t & \text { if } s \text { is odd }\end{cases}
$$

from which we conclude that

$$
\mathfrak{i}_{0}\left(\phi_{L}\right)+\mathfrak{i}_{1}\left(\phi_{L}\right) \geq \begin{cases}2^{r_{1}-1}-2^{r_{2}-1}+\cdots+2^{r_{s-1}-1}+\mathfrak{i}_{1}(\phi) & \text { if } s \text { is even, } \\ 2^{r_{1}-1}-2^{r_{2}-1}+\cdots+2^{r_{s-2}-1}+\mathfrak{i}_{1}(\phi) & \text { if } s \text { is odd. }\end{cases}
$$


But $\mathfrak{i}_{0}\left(\phi_{L}\right)+\mathfrak{i}_{1}\left(\phi_{L}\right)=\mathfrak{i}_{0}\left(\phi_{L(\phi)}\right)=\mathfrak{i}_{1}(\phi)+\mathfrak{i}_{0}\left(\left(\phi_{1}\right)_{L(\phi)}\right)$ (see the proof of Lemma 7.5), and so we have

$$
\mathfrak{i}_{0}\left(\left(\phi_{1}\right)_{L(\phi)}\right) \geq \begin{cases}2^{r_{1}-1}-2^{r_{2}-1}+\cdots+2^{r_{s-1}-1} & \text { if } s \text { is even, } \\ 2^{r_{1}-1}-2^{r_{2}-1}+\cdots+2^{r_{s-2}-1} & \text { if } s \text { is odd. }\end{cases}
$$

Either way, we see that $\mathfrak{i}_{0}\left(\left(\phi_{1}\right)_{L(\phi)}\right)>2^{r_{1}-1}-2^{r_{2}-1}+\cdots+(-1)^{s-1} 2^{r_{s}-1}=$ $\frac{1}{2}\left(\operatorname{dim} \phi-\mathfrak{i}_{1}(\phi)\right)=\frac{1}{2} \operatorname{dim} \phi_{1}$. Since $L(\phi)$ is a purely inseparable quadratic extension of $F(\phi)$, this is impossible by Lemma 2.27(5), and so the result follows.

In general, it suffices to prove Conjecture 7.3 in the case where $L$ is a finite purely inseparable extension of $F$. Proposition 7.6 shows that the $l=s$ case of the conjecture holds for degree-2 purely inseparable extensions. More generally, the proposition covers the case where $\operatorname{ndeg}\left(\phi_{L}\right)=\frac{1}{2} \operatorname{ndeg}\left(\phi_{L}\right)$. Indeed, using Lemmas 2.26 and 2.27(3), one can easily reduce this case to that of a quadratic extension.

\section{Acknowledgements}

This work was carried out while I was a visiting postdoctoral fellow at Max-PlanckInstitut für Mathematik in Bonn. I would like to thank this institution for its support and hospitality throughout the duration of my stay.

\section{References}

[EGA II 1961] A. Grothendieck, "Eléments de géométrie algébrique, II: Étude globale élémentaire de quelques classes de morphismes", Inst. Hautes Études Sci. Publ. Math. 8 (1961), 5-222. MR 0163909 Zbl 0118.36206

[Elman et al. 2008] R. Elman, N. Karpenko, and A. Merkurjev, The algebraic and geometric theory of quadratic forms, American Mathematical Society Colloquium Publications 56, American Mathematical Society, Providence, RI, 2008. MR 2427530 Zbl 1165.11042

[Haution 2013] O. Haution, "On the first Steenrod square for Chow groups", Amer. J. Math. 135:1 (2013), 53-63. MR 3022956 Zbl 1267.14012

[Haution 2015] O. Haution, "Detection by regular schemes in degree two", Algebr. Geom. 2:1 (2015), 44-61. MR 3322197 Zbl 1322.14036

[Hoffmann 1995] D. W. Hoffmann, "Isotropy of quadratic forms over the function field of a quadric", Math. Z. 220:3 (1995), 461-476. MR 1362256 Zbl 0840.11017

[Hoffmann 2004] D. W. Hoffmann, "Diagonal forms of degree $p$ in characteristic p", pp. 135-183 in Algebraic and arithmetic theory of quadratic forms, edited by R. Baeza et al., Contemp. Math. 344, American Mathematical Society, Providence, RI, 2004. MR 2058673

[Hoffmann and Laghribi 2004] D. W. Hoffmann and A. Laghribi, "Quadratic forms and Pfister neighbors in characteristic 2”, Trans. Amer. Math. Soc. 356:10 (2004), 4019-4053. MR 2058517 Zbl 1116.11020

[Hoffmann and Laghribi 2006] D. W. Hoffmann and A. Laghribi, "Isotropy of quadratic forms over the function field of a quadric in characteristic 2", J. Algebra 295:2 (2006), 362-386. MR 2194958 Zbl 1138.11012

[Izhboldin 2004] O. T. Izhboldin, "Virtual Pfister neighbors and first Witt index", pp. 131-142 in Geometric methods in the algebraic theory of quadratic forms, edited by J.-P. Tignol, Lecture Notes in Math. 1835, Springer, Berlin, 2004. MR 2066517 Zbl 1053.11033 
[Izhboldin and Vishik 2000] O. Izhboldin and A. Vishik, "Quadratic forms with absolutely maximal splitting”, pp. 103-125 in Quadratic forms and their applications (Dublin, 1999), edited by E. Bayer-Fluckiger et al., Contemp. Math. 272, American Mathematical Society, Providence, RI, 2000. MR 1803363 Zbl 0972.11017

[Karpenko 2003] N. A. Karpenko, "On the first Witt index of quadratic forms", Invent. Math. 153:2 (2003), 455-462. MR 1992018 Zbl 1032.11016

[Karpenko and Merkurjev 2003] N. Karpenko and A. Merkurjev, "Essential dimension of quadrics", Invent. Math. 153:2 (2003), 361-372. MR 1992016 Zbl 1032.11015

[Knebusch 1976] M. Knebusch, "Generic splitting of quadratic forms, I", Proc. London Math. Soc. (3) 33:1 (1976), 65-93. MR 0412101 Zbl 0351.15016

[Laghribi 2004a] A. Laghribi, "On splitting of totally singular quadratic forms", Rend. Circ. Mat. Palermo (2) 53:3 (2004), 325-336. MR 2165184 Zbl 1097.11017

[Laghribi 2004b] A. Laghribi, "Quasi-hyperbolicity of totally singular quadratic forms", pp. 237-248 in Algebraic and arithmetic theory of quadratic forms, edited by R. Baeza et al., Contemp. Math. 344, American Mathematical Society, Providence, RI, 2004. MR 2060200 Zbl 1143.11313

[Laghribi 2006] A. Laghribi, "The norm theorem for totally singular quadratic forms", Rocky Mountain J. Math. 36:2 (2006), 575-592. MR 2234821 Zbl 1142.11020

[Laghribi 2007] A. Laghribi, "Sur le déploiement des formes bilinéaires en caractéristique 2", Pacific J. Math. 232:1 (2007), 207-232. MR 2358037 Zbl 1214.11044

[Lang 2002] S. Lang, Algebra, 3rd ed., Graduate Texts in Mathematics 211, Springer, New York, 2002. MR 1878556 Zbl 0984.00001

[Merkurjev 2003] A. Merkurjev, "Steenrod operations and degree formulas", J. Reine Angew. Math. 565 (2003), 13-26. MR 2024643 Zbl 1091.14006

[Scully 2013] S. Scully, "Rational maps between quasilinear hypersurfaces", Compos. Math. 149:3 (2013), 333-355. MR 3040743 Zbl 1315.11025

[Scully 2016] S. Scully, "On the splitting of quasilinear p-forms", J. Reine Angew. Math. 713 (2016), 49-83.

[Totaro 2008] B. Totaro, "Birational geometry of quadrics in characteristic 2", J. Algebraic Geom. 17:3 (2008), 577-597. MR 2395138 Zbl 1144.11031

[Totaro 2009] B. Totaro, "Birational geometry of quadrics", Bull. Soc. Math. France 137:2 (2009), 253-276. MR 2543476 Zbl 1221.14014

[Vishik 1998] A. Vishik, "Integral motives of quadrics", preprint 1998-13, Max Planck Institute for Mathematics, 1998, available at https://www.mpim-bonn.mpg.de/preblob/286.

[Vishik 1999] A. Vishik, "Direct summands in the motives of quadrics", preprint, 1999, available at https://www.maths.nottingham.ac.uk/personal/av/Papers/dirsumE.pdf.

[Vishik 2004] A. Vishik, "Motives of quadrics with applications to the theory of quadratic forms", pp. 25-101 in Geometric methods in the algebraic theory of quadratic forms, edited by J.-P. Tignol, Lecture Notes in Math. 1835, Springer, Berlin, 2004. MR 2066515 Zbl 1047.11033

[Vishik 2011] A. Vishik, "Excellent connections in the motives of quadrics", Ann. Sci. Éc. Norm. Supér. (4) 44:1 (2011), 183-195. MR 2760197 Zbl 1223.14005

Communicated by Raman Parimala

Received 2015-08-07 Revised 2016-02-24 Accepted 2016-03-24

sscully@ualberta.ca

Department of Mathematical and Statistical Sciences, University of Alberta, Edmonton AB T6G 2G1, Canada 


\section{Algebra \& Number Theory}

msp.org/ant

\section{EDITORS}

MANAGING EDITOR

Bjorn Poonen

Massachusetts Institute of Technology

Cambridge, USA

\author{
EDITORIAL BOARD CHAIR \\ David Eisenbud \\ University of California \\ Berkeley, USA
}

BOARD OF EDITORS

$\begin{aligned} \text { Dave Benson } & \text { University of Aberdeen, Scotland } & \text { Susan Montgomery } & \text { University of Southern California, USA } \\ \text { Richard E. Borcherds } & \text { University of California, Berkeley, USA } & \text { Shigefumi Mori } & \text { RIMS, Kyoto University, Japan } \\ \text { John H. Coates } & \text { University of Cambridge, UK } & \text { Raman Parimala } & \text { Emory University, USA } \\ \text { J-L. Colliot-Thélène } & \text { CNRS, Université Paris-Sud, France } & \text { Jonathan Pila } & \text { University of Oxford, UK } \\ \text { Brian D. Conrad } & \text { Stanford University, USA } & \text { Anand Pillay } & \text { University of Notre Dame, USA } \\ \text { Hélène Esnault } & \text { Freie Universität Berlin, Germany } & \text { Victor Reiner } & \text { University of Minnesota, USA } \\ \text { Hubert Flenner } & \text { Ruhr-Universität, Germany } & \text { Peter Sarnak } & \text { Princeton University, USA } \\ \text { Sergey Fomin } & \text { University of Michigan, USA } & \text { Joseph H. Silverman } & \text { Brown University, USA } \\ \text { Edward Frenkel } & \text { University of California, Berkeley, USA } & \text { Michael Singer } & \text { North Carolina State University, USA } \\ \text { Andrew Granville } & \text { Université de Montréal, Canada } & \text { Vasudevan Srinivas } & \text { Tata Inst. of Fund. Research, India } \\ \text { Joseph Gubeladze } & \text { San Francisco State University, USA } & \text { J. Toby Stafford } & \text { University of Michigan, USA } \\ \text { Roger Heath-Brown } & \text { Oxford University, UK } & \text { Ravi Vakil } & \text { Stanford University, USA } \\ \text { Craig Huneke } & \text { University of Virginia, USA } & \text { Michel van den Bergh } & \text { Hasselt University, Belgium } \\ \text { Kiran S. Kedlaya } & \text { Univ. of California, San Diego, USA } & \text { Marie-France Vignéras } & \text { Université Paris VII, France } \\ \text { János Kollár } & \text { Princeton University, USA } & \text { Kei-Ichi Watanabe } & \text { Nihon University, Japan } \\ \text { Yuri Manin } & \text { Northwestern University, USA } & \text { Efim Zelmanov } & \text { University of California, San Diego, USA } \\ \text { Philippe Michel } & \text { École Polytechnique Fédérale de Lausanne } & \text { Shou-Wu Zhang } & \text { Princeton University, USA }\end{aligned}$

PRODUCTION

production@msp.org

Silvio Levy, Scientific Editor

See inside back cover or msp.org/ant for submission instructions.

The subscription price for 2016 is US $\$ 290$ /year for the electronic version, and $\$ 485 /$ year (+\$55, if shipping outside the US) for print and electronic. Subscriptions, requests for back issues and changes of subscribers address should be sent to MSP.

Algebra \& Number Theory (ISSN 1944-7833 electronic, 1937-0652 printed) at Mathematical Sciences Publishers, 798 Evans Hall \#3840, c/o University of California, Berkeley, CA 94720-3840 is published continuously online. Periodical rate postage paid at Berkeley, CA 94704, and additional mailing offices.

ANT peer review and production are managed by EditFLow ${ }^{\circledR}$ from MSP.

\section{PUBLISHED BY}

- mathematical sciences publishers

nonprofit scientific publishing

http://msp.org/

() 2016 Mathematical Sciences Publishers 


\section{Algebra \& Number Theory}

Volume $10 \quad$ No. $5 \quad 2016$

Conjugacy classes of special automorphisms of the affine spaces

JÉRÉMY BLANC

Inversion of adjunction for rational and Du Bois pairs

SÁNDOR J. KOVÁCS and KARL SCHWEDE

Hochschild cohomology commutes with adic completion

LIRAN SHAUL

Bifurcations, intersections, and heights

LAURA DEMARCO

RANKEyA DATTA and KaREN E. SMITH

1091

Hoffmann's conjecture for totally singular forms of prime degree

STEPHEN SCULLY 